\title{
Dynamical fixed points in holography
}

\author{
Alex Buchel \\ Department of Physics and Astronomy, University of Western Ontario, \\ London, ON N6A 5B\%, Canada \\ Perimeter Institute for Theoretical Physics, \\ Waterloo, ON N2J 2W9, Canada \\ E-mail: abuchel@uwo.ca
}

ABSTRACT: Typically, an interactive system evolves towards thermal equilibrium, with hydrodynamics representing a universal framework for its late-time dynamics. Classification of the dynamical fixed points (DFPs) of a driven Quantum Field Theory (with time dependent coupling constants, masses, external background fields, etc.) is unknown. We use holographic framework to analyze such fixed points in one example of strongly coupled gauge theory, driven by homogeneous and isotropic expansion of the background metric - equivalently, a late-time dynamics of the corresponding QFT in Friedmann-LemaitreRobertson-Walker Universe. We identify DFPs that are perturbatively stable, and those that are perturbatively unstable, computing the spectrum of the quasinormal modes in the corresponding holographic dual. We further demonstrate that a stable DFP can be unstable non-perturbatively, and explain the role of the entanglement entropy density as a litmus test for a non-perturbative stability. Finally, we demonstrated that a driven evolution might not have a fixed point at all: the entanglement entropy density of a system can grow without bounds.

KEYwords: AdS-CFT Correspondence, Gauge-Gravity Correspondence

ArXIV EPRINT: 2111.04122 


\section{Contents}

1 Introduction and summary $\quad 2$

2 Holographic model 5

$\begin{array}{llr}3 & \text { Results } & 7\end{array}$

$3.1 \mathbb{Z}_{2}^{\chi}$-symmetric DFP - $\mathrm{DFP}_{s} \quad 7$

3.2 DFP with spontaneously broken $\mathbb{Z}_{2}^{\chi}$ symmetry $-\mathrm{DFP}_{b} \quad 9$

3.3 Dynamics of the holographic model with linearized $\mathbb{Z}_{2}^{\chi}$ sector 11

$\begin{array}{lll}3.4 \mathrm{DFP}_{s} \text { or } \mathrm{DFP}_{b} \text { ? } & 13\end{array}$

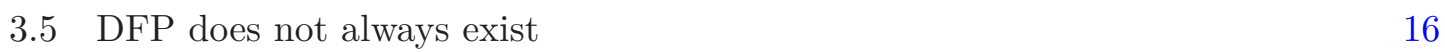

$\begin{array}{ll}3.6 & \text { Dynamics of } \mathrm{DFP}_{b} \\ \end{array}$

4 Conclusions $\quad 16$

A Holographic EOMs, the boundary asymptotics and the renormalization 18

B $\mathbb{Z}_{\mathbf{2}}$-symmetric DFP and its fluctuations $\quad 21$

B.1 $\mathbb{Z}_{2}^{\chi}$-symmetric DFP 21

B.2 Fluctuations about $\mathbb{Z}_{2}^{\chi}$-symmetric DFP 24

C DFP with spontaneously broken $\mathbb{Z}_{2}$ symmetry and its fluctuations $\quad 25$

$\begin{array}{lll}\text { C.1 } & \mathbb{Z}_{2}^{\chi} \text {-broken DFP } & 25\end{array}$

$\begin{array}{ll}\text { C.2 } & \text { Fluctuations about } \mathbb{Z}_{2}^{\chi} \text {-broken DFP } \\ & 27\end{array}$

D Dynamical entropy and the entanglement entropy of a DFP 29

$\begin{array}{ll}\text { D.1 Dynamical entropy from the holographic dual } & 29\end{array}$

D.2 Entanglement entropy density of a DFP 30

$\begin{array}{ll}\text { E Numerical setup } & 31\end{array}$

E.1 Field redefinitions and the code equations 31

E.2 Apparent horizon and the boundary condition for $a \quad 34$

E.3 Initial conditions 34

E.4 DFP with spontaneously broken $\mathbb{Z}_{2}$ symmetry as an initial condition $\quad 35$

$\begin{array}{lll}\text { E.5 Convergence tests } 36 & 36\end{array}$ 


\section{Introduction and summary}

Thermodynamic equilibrium is an internal state of a system without the net macroscopic flow of matter or energy. This equilibrium state is characterized by few time-independent, constant over the material sample, properties: the energy density $\mathcal{E}_{\text {eq }}$, the pressure $P_{\text {eq }}$, the entropy density $s_{\mathrm{eq}}$, the temperature $T$, etc. Isolated interacting quantum systems typically ${ }^{1}$ reach thermodynamic equilibrium at late times of their dynamical evolution $[3,4]$ :

$$
\lim _{\tau \rightarrow \infty} T_{\mu \nu}(\tau, \boldsymbol{x})=\operatorname{diag}\left(\mathcal{E}_{\mathrm{eq}}, P_{\mathrm{eq}}, \cdots P_{\mathrm{eq}}\right)
$$

where $T_{\mu \nu}$ are the component of the stress-energy tensor of the system at time $\tau$ and the spatial location $\boldsymbol{x}$. Moreover, the approach to thermal equilibrium, whenever the spacetime gradients of the local thermodynamic observables are small compare to the energy scale set by the local temperature, is universally governed by the hydrodynamics [5]. For example, in the absence of conserved charges, given a time-like unit vector $u^{\mu}=u^{\mu}(\tau, \boldsymbol{x})$, the Landau frame stress-energy tensor decomposes as

$$
T^{\mu \nu}=\mathcal{E} u^{\mu} u^{\nu}+P \Delta^{\mu \nu}+\mathcal{T}^{\mu \nu}, \quad \mathcal{T}^{\mu \nu}=-\eta \sigma^{\mu \nu}-\zeta \Delta^{\mu \nu}(\nabla \cdot u),
$$

where $g_{\mu \nu}$ is the background metric, $\Delta^{\mu \nu} \equiv g^{\mu \nu}+u^{\mu} u^{\nu}, \sigma^{\mu \nu}$ is some symmetric transverse traceless tensor of the first derivatives of $u^{\mu}$, and $\mathcal{E}=\mathcal{E}(\tau, \boldsymbol{x})$ is the local energy density. The shear $\eta$ and the bulk $\zeta$ viscosities are functions of the local energy density, and can be computed from the equilibrium two-point correlation functions of the stress-energy tensor. The local pressure $P=P_{\text {eq }}(\mathcal{E})$ is determined by the equilibrium equation of state, and the local entropy density $s=s(\mathcal{E})$ and the temperature $T=T(\mathcal{E})$ follow from the basic laws of the equilibrium thermodynamics:

$$
\mathcal{E}+P=s T, \quad d \mathcal{E}=T d s .
$$

The second law of thermodynamics postulates that the divergence of the entropy current $\mathcal{S}^{\mu}$ is non-negative under physical processes

$$
\nabla \cdot \mathcal{S} \geq 0
$$

There is no first-principle definition of $\mathcal{S}^{\mu}$ far from equilibrium; in the hydrodynamic approximation, i.e., to the first-order in the gradients of the local fluid velocity $u^{\mu},[6,7]$

$$
\mathcal{S}^{\mu}=s u^{\mu}-\frac{1}{T} \mathcal{T}^{\mu \nu} u_{\nu}+\mathcal{O}\left(\partial^{2} u\right) .
$$

Conservation of the stress-energy tensor (1.2) then implies

$$
T \nabla \cdot \mathcal{S}=\zeta(\nabla \cdot u)^{2}+\frac{\eta}{2} \sigma_{\mu \nu} \sigma^{\mu \nu}+\mathcal{O}\left(\partial^{3} u\right),
$$

which is manifestly non-negative, provided the viscosities are positive. At thermal equilibrium the divergence of the entropy current (1.4) vanishes.

\footnotetext{
${ }^{1}$ Some of the counterexamples are the integrable systems, Fermi-Pasta-Ulam-Tsingou problem [1], and gravitational collapse in AdS [2].
} 
We can now provide a formal definition of a dynamical fixed point (DFP):

A Dynamical Fixed Point is an internal state of a quantum field theory with spatially homogeneous and time-independent one-point correlation functions of its stress energy tensor $T^{\mu \nu}$, and (possibly additional) set of gauge-invariant local operators $\left\{\mathcal{O}_{i}\right\}$,

and

strictly positive divergence of the entropy current at late-times:

$$
\lim _{\tau \rightarrow \infty}(\nabla \cdot \mathcal{S})>0
$$

Note that apart from the requirement of the strictly non-zero entropy production rate at late times, characteristics of a DFP coincide with that of the thermodynamic equilibrium. The late-time entropy production can arise when a QFT is driven externally by varying in time the coupling constants of the relevant operators, masses, background fields (e.g., the space-time metric). In this paper we study DFPs of a QFT in a cosmological background, the de Sitter background spacetime in particular.

DFP classification necessitates the assignment of the entropy current $\mathcal{S}^{\mu}$ to a system, defined for its arbitrary far-from-equilibrium configurations. From the QFT perspective, this is an unsolved problem - the progress can be made though for theories with a dual holographic gravitational descriptions $[8,9]$. In variety of holographic models [10-12] it was rigorously proven that the comoving gravitational entropy density of the apparent horizon, associated with some natural observer (the spatial slicing), can not decrease with time,

$$
\frac{d s_{\text {comoving }}^{A H}}{d \tau} \geq 0
$$

It is natural to identify this gravitational entropy density $s_{\text {comoving }}^{A H}$ as the comoving entropy density of a boundary QFT, $s_{\text {comoving: }}$

$$
s_{\text {comoving }} \equiv s_{\text {comoving }}^{A H} .
$$

If $a(\tau)$ is a scale factor of the QFT background $d$-dimensional Friedmann-LemaitreRobertson-Walker (FLRW) Universe

$$
d s_{d}^{2}=-d \tau^{2}+a(\tau)^{2} d \boldsymbol{x}^{2},
$$

the comoving $s_{\text {comoving }}$ and the physical $s$ entropy densities are related as

$$
s_{\text {comoving }}(\tau)=a(\tau)^{d-1} s(\tau) .
$$

Thus, is we define the entropy current as

$$
\mathcal{S}^{\mu}=s(\tau) u^{\mu}, \quad u^{\mu} \equiv(1,0, \cdots, 0),
$$

we find that

$$
\nabla \cdot \mathcal{S}=\frac{1}{a(\tau)^{d-1}} \frac{d}{d \tau}\left(a(\tau)^{d-1} s(\tau)\right)=\frac{1}{a(\tau)^{d-1}} \frac{d}{d \tau} s_{\text {comoving }}(\tau) \geq 0,
$$


due to (1.7). In [13], the late-time, $\tau \rightarrow \infty$, limit of the physical entropy $s(\tau)$, provided this limit exists, was called the vacuum entanglement entropy (VEE) density

$$
\lim _{\tau \rightarrow \infty} s(\tau)=s_{\text {ent }}
$$

Specializing to de Sitter Universe, i.e., $a(\tau)=e^{H \tau}$, we determine the late time entropy production rate as

$$
\lim _{\tau \rightarrow \infty}(\nabla \cdot \mathcal{S})=(d-1) H s_{\text {ent }} .
$$

Due to (1.7), the VEE is nonnegative; if it is strictly positive, as in $[11,12,14]$, it characterizes a DFP.

Note that an interacting conformal field theory driven by (1.9) can not have a DFP. Indeed, a $\mathrm{CFT}_{d}$ dynamics in (1.9) by a conformal transformation is equivalent to a dynamics in Minkowski space-time. Furthermore, the entropy production rate associated with the dynamics of the apparent horizon is invariant under the conformal transformations [11]. Since a closed interacting theory in Minkowski space-time is expected to equilibrate,

$$
\lim _{\tau \rightarrow \infty} \nabla \cdot \mathcal{S}=0 .
$$

As we explicitly demonstrate in the paper:

- A holographic non-conformal QFT can have several DFPs, depending on the ratio of its mass-scale $\Lambda$ and the Hubble constant $H$.

- As in [12], distinct DFPs are characterized by a pattern of the spontaneous global symmetry breaking - importantly, they have different vacuum entanglement entropy densities.

- We present examples of DFPs that are perturbatively stable, and those that are unstable to fluctuations of energy density and/or the global symmetry order parameter. $^{2}$

- Following (1.14), when several DFPs are present given $\frac{\Lambda}{H}$, the one with the larger $s_{\text {ent }}$ would lead at late times to a larger comoving entropy production rate. In our model, DFPs with the larger VEE density have spontaneous symmetry breaking. ${ }^{3}$ The symmetry preserving DFP is perturbatively stable to fluctuations of the symmetry breaking order parameter — thus, to reach "more entropic" symmetry broken DFP, one needs a large enough amplitude of the symmetry breaking fluctuations. In other words, a perturbatively stable DFP can be non-perturbatively unstable.

- We demonstrate that a DFP of a non-conformal QFT in de Sitter need not exist, at least within controllable holographic framework: we identify $\frac{\Lambda}{H}$ parameter range

\footnotetext{
${ }^{2}$ This might have implications to cosmological model building: instabilities in late-time de Sitter cosmology of a strongly coupled gauge theory can lead to observable imprints in the cosmic microwave background.

${ }^{3}$ That a global symmetry broken DFP always has a larger $s_{\text {ent }}$ is not true in general, see [12] for a counterexample.
} 
where initially arbitrarily small amplitude symmetry breaking fluctuations evolve the (initially smooth) dual gravitational geometry to a naked singularity. The physical entropy density $s$ growth without bounds, implying that the limit (1.13) does not exist.

The rest of the paper is organized as follows. In the next section we introduce our holographic model - it was been extensively studied in holography in the past [14-18]. While the model is a 'toy' model of the holographic correspondence, it has top-down holographic cousins, i.e., [11, 12], that share some similarities with respect to DFPs. The advantage of the model is that it allows for a relatively simple numerical simulation, enabling the study of DFPs as late-time attractors of its dynamical evolution. Numerical codes used present an adaptation of the codes deployed in [17] and [14]. To keep the discussion self-contained, we review the adapted codes in appendix E. Results are presented in section 3, with minimum of technical details: the holographic dictionary of the model is covered in appendix A; appendices B and C discuss symmetric and symmetry-broken DFPs of the model, including the details necessary to compute the spectra of linearized fluctuations; appendix D contains the proof of (1.7) for our model, ${ }^{4}$ as well as the explicit expression for VEE densities of the model DFPs. Finally, conclusions and future directions are covered in section 4.

The work reported here is heavily numerical. Convergence tests of the numerical simulations are discussed in appendix E.5. Numerical construction of DFPs and the computations of the spectra of the linearized fluctuations are done using Wolfram Mathematica, while the evolution codes are implemented in FORTRAN. The fact that perturbatively stable DFPs are attractors of the late-time dynamics, including the approach rate to a DFP (as set by the lowest quasinormal mode (QNM) frequency of the linearized fluctuations), is a highly nontrivial cross check between the two numerical platforms. Likewise, there is an excellent agreement between the growth rate of unstable (small amplitude) fluctuations in a simulation, with the corresponding frequency of the unstable QNM of the DFP. Another nontrivial consistency check on numerics is the high accuracy agreement between the two different expressions for the vacuum entanglement entropy densities of various DFPs, see appendix D.2.

\section{Holographic model}

We restrict our attention to a simple holographic toy model of a $d=2+1$-dimensional massive $Q F T_{3}$ with the effective dual gravitational action: ${ }^{5}$

$$
\begin{aligned}
& S_{4}=S_{\mathrm{CFT}}+S_{r}+S_{i}=\frac{1}{2 \kappa^{2}} \int_{\mathcal{M}_{4}} d x^{4} \sqrt{-\gamma}\left[\mathcal{L}_{\mathrm{CFT}}+\mathcal{L}_{r}+\mathcal{L}_{i}\right], \\
& \mathcal{L}_{\mathrm{CFT}}=R+6, \quad \mathcal{L}_{r}=-\frac{1}{2}(\nabla \phi)^{2}+\phi^{2}, \quad \mathcal{L}_{i}=-\frac{1}{2}(\nabla \chi)^{2}-2 \chi^{2}-g \phi^{2} \chi^{2} ;
\end{aligned}
$$

where we split the action into (a holographic dual to) a $C F T_{3}$ part $S_{\mathrm{CFT}}$; its deformation by a relevant operator $\mathcal{O}_{\phi}$,

$$
L^{2} m_{\phi}^{2}=-2=\Delta_{\phi}\left(\Delta_{\phi}-d\right),
$$

\footnotetext{
${ }^{4}$ It would be nice to prove the relevant apparent horizon theorem in full generality, rather than on case-by-case basis as done here, and in earlier work $[10-12,14]$.

${ }^{5}$ We set the radius $L$ of an asymptotic $A d S_{4}$ geometry to unity.
} 
and a sector $S_{i}$ involving an irrelevant operator $\mathcal{O}_{\chi}$,

$$
L^{2} m_{\chi}^{2}=+4=\Delta_{\chi}\left(\Delta_{\chi}-d\right),
$$

along with its mixing with $\mathcal{O}_{\phi}$ under the renormalization-group flow, represented by a bulk interaction $-g \phi^{2} \chi^{2}$. We take bulk quantization so that the scaling dimension of $\mathcal{O}_{\phi}$ is $\Delta_{\phi}=2$; the scaling dimension of $\mathcal{O}_{\chi}$ is $\Delta_{\chi}=4$. In order to have asymptotically $A d S_{4}$ solutions, we assume that only the normalizable mode of $\mathcal{O}_{\chi}$ is nonzero near the boundary. The four dimensional gravitational constant $\kappa$ is related to the ultraviolet (UV) conformal fixed point $C F T_{3}$ central charge $c$ as

$$
c=\frac{192}{\kappa^{2}} .
$$

The gravitational action (2.1) has $\mathbb{Z}_{2}^{\phi} \times \mathbb{Z}_{2}^{\chi}$ discrete symmetry that acts as a parity transformation on the scalar fields $\phi$ and $\chi$ correspondingly. The discrete symmetry $\phi \leftrightarrow$ $-\phi$ is explicitly broken by the relevant deformation of the CFT,

$$
\mathcal{H}_{\mathrm{CFT}} \rightarrow \mathcal{H}_{\mathrm{CFT}}+\Lambda \mathcal{O}_{\phi},
$$

with $\Lambda$ being the deformation mass scale, while the $\chi \leftrightarrow-\chi$ symmetry is broken spontaneously whenever $\mathcal{O}_{\chi} \neq 0$. We are interested in the holographic dynamics of the boundary theory (2.1), driven by the homogeneous and isotropic expansion of its background metric

$$
d s_{\partial \mathcal{M}_{4}}^{2}=-d \tau^{2}+e^{2 H \tau}\left(d x_{1}^{2}+d x_{2}^{2}\right)
$$

with a constant Hubble parameter $H$.

A generic state of the boundary field theory with a gravitational dual (2.1), homogeneous and isotropic in the spatial boundary coordinates $\boldsymbol{x}=\left\{x_{1}, x_{2}\right\}$, leads to a bulk gravitational metric ansatz ${ }^{6}$

$$
d s_{4}^{2}=2 d \tau(d r-A d \tau)+\Sigma^{2} d \boldsymbol{x}^{2},
$$

with the warp factors $A, \Sigma$ as well as the bulk scalar $\phi$ and $\chi$ depending only on $\{t, r\}$. We collect equations of motion obtained from (2.1), within the bulk ansatz (2.8), in appendix A. Appendix A explains how one extracts the $Q F T_{3}$ observables: the energy density $\mathcal{E}(t)$, the pressure $P(t)$, and the expectation values of the operators $\mathcal{O}_{\phi}(t)$ and $\mathcal{O}_{\chi}(t)$ from the holographic bulk dynamics of the given initial state.

Another important observable is the non-equilibrium entropy density $s(\tau)$. This physical entropy density should not to be confused with the comoving entropy density, corresponding to the boundary QFT background space-time expansion (2.7):

$$
s_{\text {comoving }}(\tau)=e^{2 H \tau} s(\tau) .
$$

A holographic characteristic formulation of the boundary dynamics (2.8) implies a preferred spatial slicing (a preferred observer) — we associate the comoving non-equilibrium entropy density with the comoving Bekenstein-Hawking entropy density of the corresponding apparent horizon, see appendix D. As defined, the comoving entropy density has the following properties:

\footnotetext{
${ }^{6}$ We use the characteristic formulation of the holographic dynamics [19].
} 

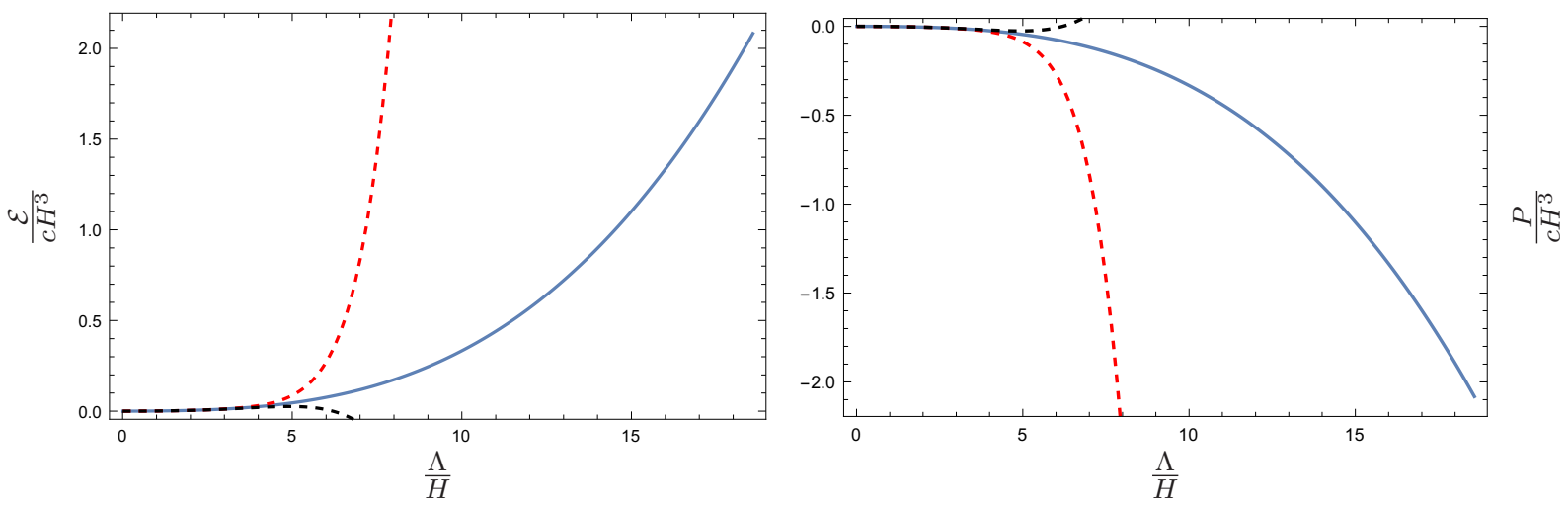

Figure 1. The energy density $\mathcal{E}$ (the left panel) and the pressure $P$ (the right panel) of the $\mathbb{Z}_{2}^{\chi}$ symmetric DFP (solid curves). The black/red dashed curves present the (successive) perturbative in the limit $\frac{\Lambda}{H} \rightarrow 0$ approximations to the observables.

- It can only grow with time (see [10-12] and section D.1),

$$
\frac{d s_{\text {comoving }}}{d \tau} \geq 0
$$

- It coincides with the thermal entropy density at equilibrium.

- In the adiabatic approximation, i.e., $H \ll \Lambda$, and for initial configurations close to thermal equilibrium, the comoving entropy density production rate $[5,20]$ is governed by the hydrodynamics [21]:

$$
\frac{d}{d \tau} \ln s_{\text {comoving }} \approx(\nabla \cdot u)^{2} \frac{\zeta}{\mathcal{E}+P}=(2 H)^{2} \frac{\zeta}{\mathcal{E}+P},
$$

where $\zeta$ is the bulk viscosity of the boundary QFT, and we identified the homogeneous and isotropic expansion of the background space-time (2.7) with the locally static hydrodynamic expansion $u^{i}=(1,0,0)$ with a nonzero expansion rate $\Theta \equiv \nabla_{i} u^{i}=2 H$.

An initial state of a QFT, for a specified driving rate $\frac{H}{\Lambda}$, will evolve according to the gravitational dynamics as explained in appendix A. The late-time $H \tau \rightarrow \infty$ state is a DFP provided the limit (1.13) exists.

\section{Results}

We choose

$$
g=-100 .
$$

All the results reported depend on the choice of this parameter in the effective action (2.2).

\section{$3.1 \mathbb{Z}_{2}^{\chi}$-symmetric DFP - DFP $_{s}$}

The effective action (2.1) can be consistently truncated to a $\mathbb{Z}_{2}^{\chi}$-symmetric sector, i.e., setting

$$
\chi(\tau, r) \equiv 0 .
$$



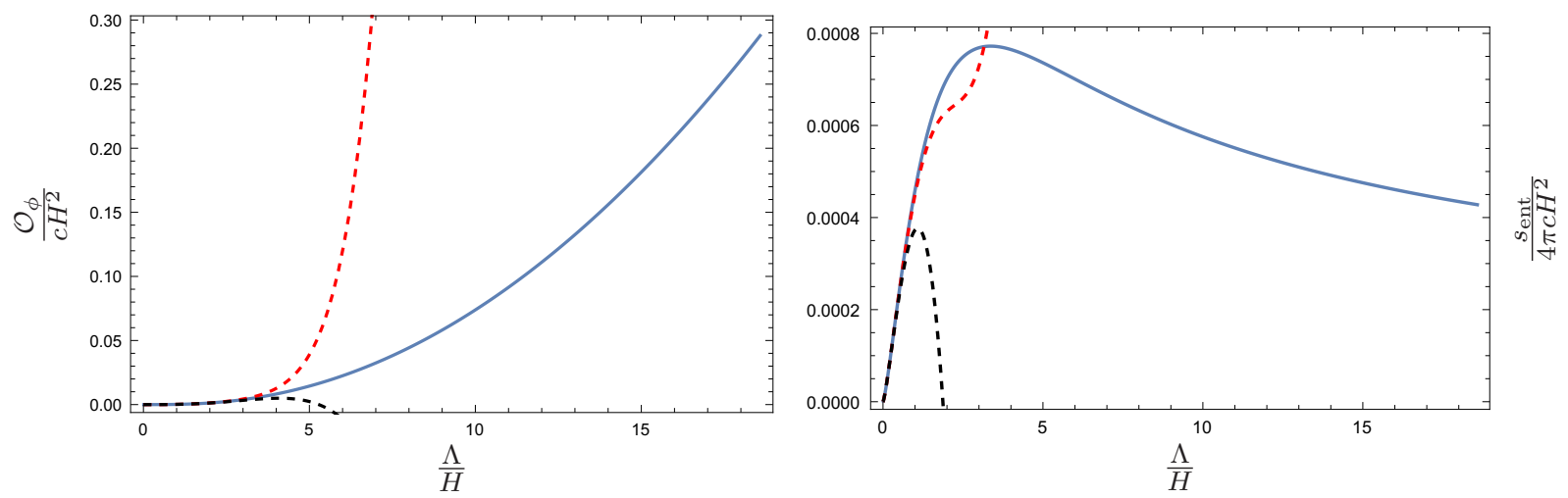

Figure 2. The expectation value $\mathcal{O}_{\phi}$ (the left panel) and the vacuum entanglement entropy density $s_{\text {ent }}$ (the right panel) of the $\mathbb{Z}_{2}^{\chi}$-symmetric DFP (solid curves). The black/red dashed curves present the (successive) perturbative in the limit $\frac{\Lambda}{H} \rightarrow 0$ approximations to the observables.
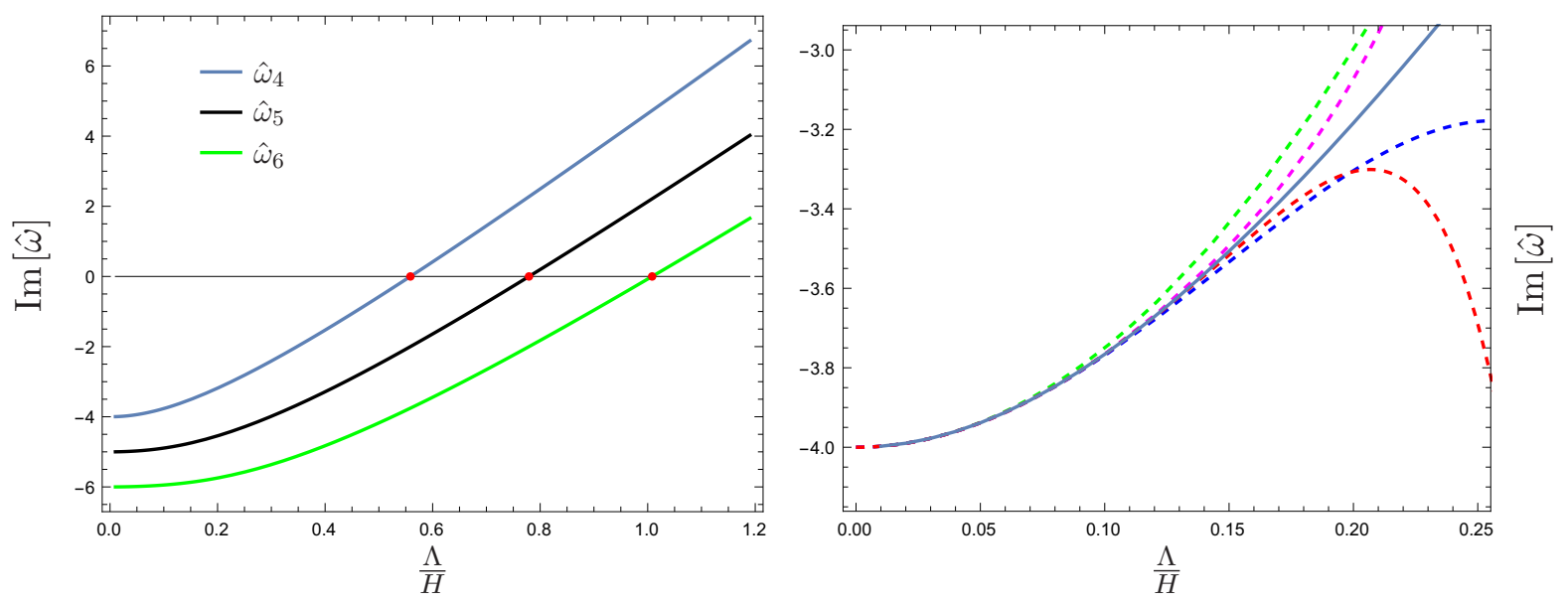

Figure 3. Spectra $\hat{\omega}_{n}=\hat{\omega}_{n}(\Lambda / H)$ of $\mathbb{Z}_{2}^{\chi}$ symmetry breaking fluctuations about the $\mathrm{DFP}_{s}$. The red dots indicate the onset of the instability, see (3.5). In the limit $\frac{\Lambda}{H} \rightarrow 0$, the $\hat{\omega}_{n}$ spectra can be computed analytically. The right panel shows $\hat{\omega}_{4}$ (the solid curve), and the dashed curves are the successive perturbative approximations, see (B.22).

This truncation has been studied extensively in [14]. In appendix B.1 we present the technical details, pertinent to the construction of the $\mathrm{DFP}_{s}$. This DFP exists for

$$
\frac{|\Lambda|}{H}>0
$$

The $\mathbb{Z}_{2}^{\phi}$ symmetry is explicitly broken by $\Lambda \neq 0$, the results presented are invariant under $\Lambda \leftrightarrow-\Lambda$; we assume $\Lambda>0$.

In figures 1 and 2 we present the results for the energy density $\mathcal{E}$, the pressure $P$, the expectation value of the operator $\mathcal{O}_{\phi}$, and the VEE density of the $\mathrm{DFP}_{s}$, as one varies $\frac{\Lambda}{H}$ (the solid curves). In the limit $\frac{\Lambda}{H} \rightarrow 0$ all the observables can be computed analytically see (B.16) and (D.13). The red/black dashed curves represent the highest/next-to-highest (computed) perturbative approximations to the given observable. 

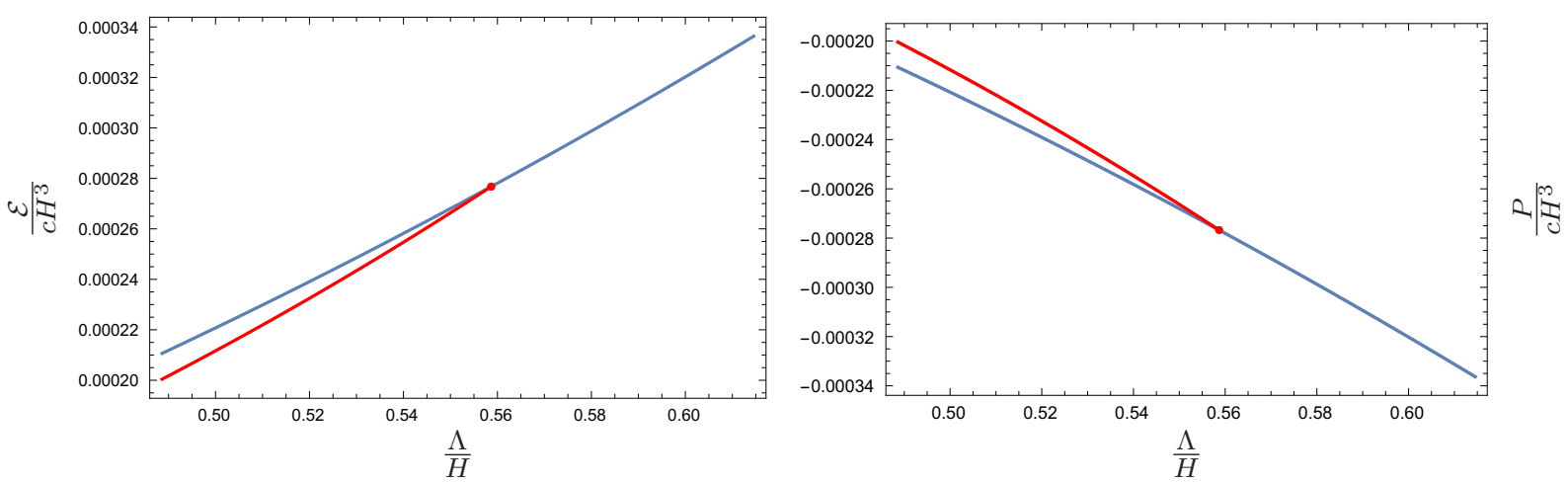

Figure 4. The energy density $\mathcal{E}$ (the left panel) and the pressure $P$ (the right panel) of $\mathbb{Z}_{2}^{\chi}$ symmetric DFP (the blue curves) and the symmetry broken DFP (the red curves). $\mathrm{DFP}_{b}$ exists only above certain critical value of the Hubble constant, see (3.6).

As explored in [14], $\mathrm{DFP}_{s}$ is perturbatively stable under the strict truncation (3.2). In appendix B.2 we study the spectrum of $\mathbb{Z}_{2}^{\chi}$ symmetry breaking fluctuations about the $\mathrm{DFP}_{s}$. The results are collected in figure 3. In the limit $\frac{\Lambda}{H} \rightarrow 0$ the spectrum of the QNMs can be computed analytically — see (B.22) for the $\hat{\omega}_{4}$ mode (the solid curve in the right panel; the dashed curves represent the successive perturbative approximations). While these linearized fluctuations are stable for small enough $\frac{\Lambda}{H}$, they all eventually become unstable (solid curves, the left panel):

$$
\begin{array}{lll}
\operatorname{Im}\left[\hat{\omega}_{4}\right]>0 & \Longrightarrow & \frac{\Lambda}{H}>p_{1,4}^{\text {crit }} \equiv 0.55867(6) ; \\
\operatorname{Im}\left[\hat{\omega}_{5}\right]>0 & \Longrightarrow & \frac{\Lambda}{H}>p_{1,5}^{\text {crit }} \equiv 0.77919(5) ; \\
\operatorname{Im}\left[\hat{\omega}_{6}\right]>0 & \Longrightarrow & \frac{\Lambda}{H}>p_{1,6}^{\text {crit }} \equiv 1.0082(4) .
\end{array}
$$

The red dots indicate the onset of the instability

$$
\left.\operatorname{Im}\left[\hat{\omega}_{n}\right]\right|_{\frac{\Lambda}{H}=p_{1, n}^{\text {crit }}}=0 .
$$

\subsection{DFP with spontaneously broken $\mathbb{Z}_{2}^{\chi}$ symmetry - $\mathrm{DFP}_{b}$}

The onset of the $\mathbb{Z}_{2}^{\chi}$ spontaneous symmetry breaking instability associated with the QNM $\hat{\omega}_{n}$ about $\mathrm{DFP}_{s}$, see (3.5), represent a coexistence point of $\mathrm{DFP}_{s}$ and a new, symmetry broken, dynamical fixed point $\mathrm{DFP}_{b}^{n}$. In appendix C.1 we collect the technical details, pertinent to the construction of the $\mathrm{DFP}_{b}^{n}$. We present the results only ${ }^{7}$ for $\mathrm{DFP}_{b}^{4}$, henceforth denoted simply as $\mathrm{DFP}_{b}$.

We constructed $\mathrm{DFP}_{b}$ for a fairy narrow range

$$
\frac{\Lambda}{H} \in\left(\approx 0.4886, p_{1,4}^{\text {crit }}=0.55867(6)\right]
$$

\footnotetext{
${ }^{7}$ It is straightforward to construct higher- $n$ dynamical fixed points — we leave the detailed analysis for the future.
} 

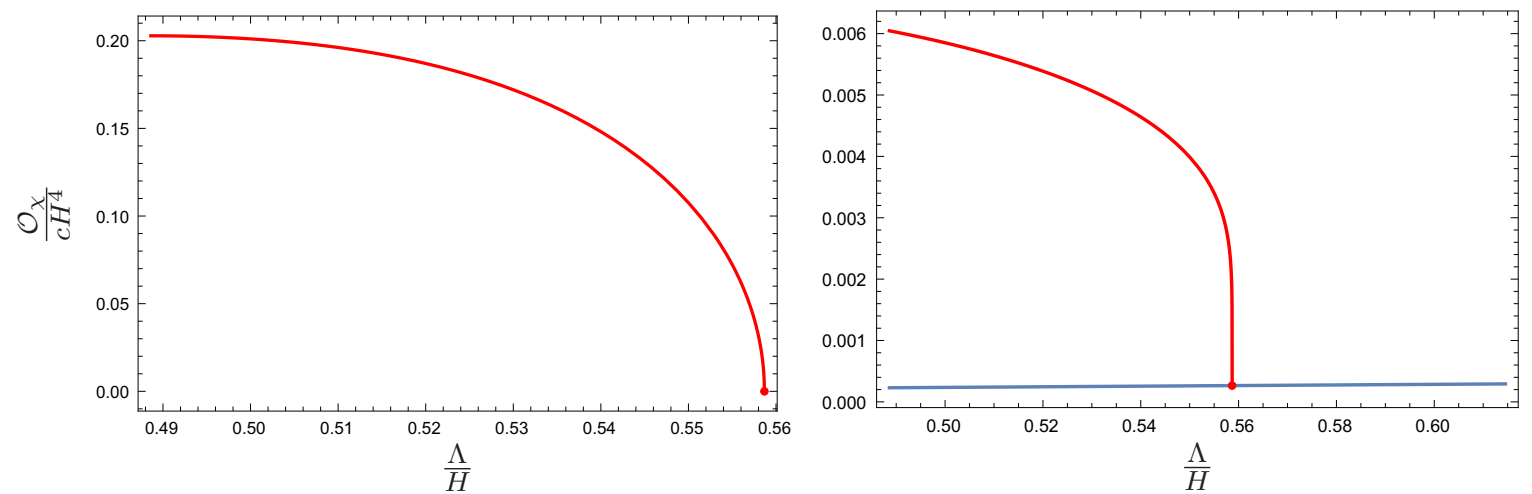

Figure 5. The left panel: the expectation value of the $\mathbb{Z}_{2}^{\chi}$ symmetry breaking order parameter $\mathcal{O}_{\chi}$. The right panel: the vacuum entanglement entropies of the $\mathrm{DFP}_{s}$ (the blue curve) and the $\mathrm{DFP}_{b}$ (the red curve).
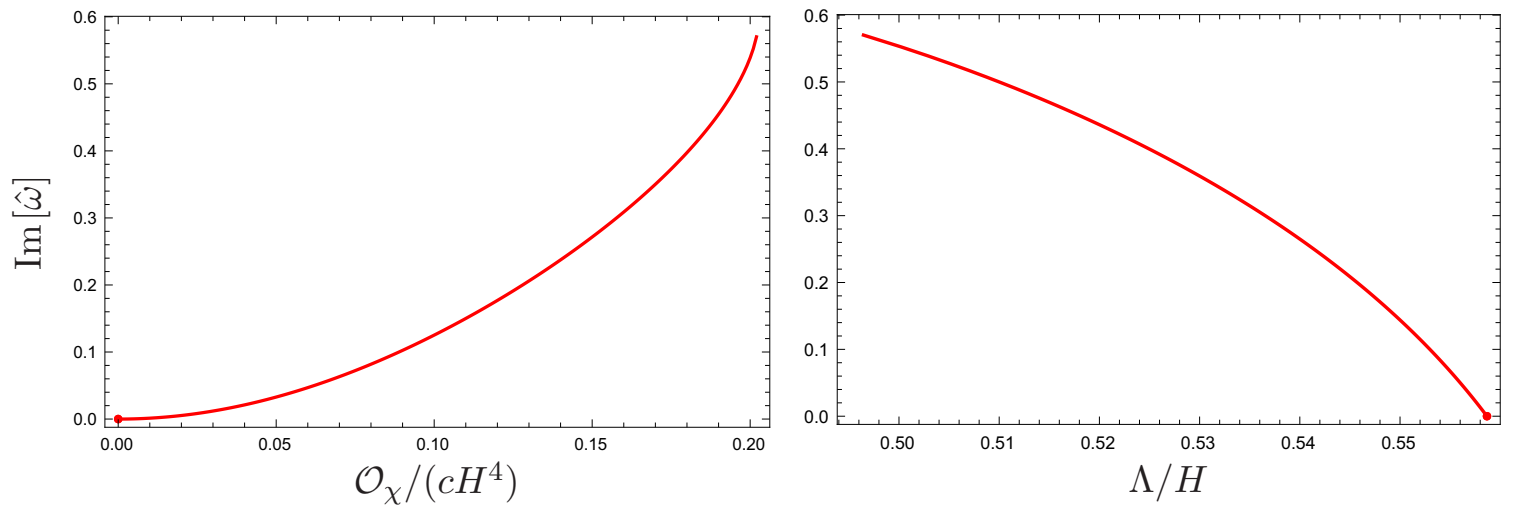

Figure 6. The $\mathrm{DFP}_{b}$ is perturbatively unstable. The left panel: $\hat{\omega}$ of the unstable QNM as a function of the symmetry breaking order parameter in the $\mathrm{DFP}_{b}$. The right panel: $\hat{\omega}$ as a function of $\frac{\Lambda}{H}$ of $\mathrm{DFP}_{b}$.

The upper bound is set by the onset of the instability due to $\hat{\omega}_{4}$, see (3.4); and the lower bound is due to the limitations of the numerical codes we used. We determined that as $H$ increases, the Kretschmann scalar evaluated at the apparent horizon grows - it increases over the range (3.6) by a factor of $\approx 66$, making the dual supergravity approximation less reliable. There must exist an obstruction for a $\operatorname{DFP}_{b}$ as $\frac{\Lambda}{H} \rightarrow 0$ : in this limit the model is conformal. Note that the symmetry broken DFP exists for $H>\frac{1}{p_{1,4}^{\text {crit }}} \Lambda$ - this is similar to the DFP of the cascading gauge theory with spontaneously broken chiral symmetry, which also exists for $H>H_{\min } \propto \Lambda[12]$.

In figure 4 we compare the energy density and the pressure of the $\mathrm{DFP}_{s}$ (the blue curves) and the $\mathrm{DFP}_{b}$ (the red curves). While the stress-energy tensor of the model is renormalization scheme dependent, see (A.11) and (A.12), the comparison is meaningful in a fixed scheme: notice that the $\mathrm{DFP}_{b}$ has the lower energy density, and the higher pressure.

In the left panel of figure 5 we present the expectation value of the order parameter $\mathcal{O}_{\chi}$ for the spontaneous breaking of $\mathbb{Z}_{2}^{\chi}$ symmetry in the $\mathrm{DFP}_{b}$. In the right panel we compare the VEE densities $s_{\text {ent }}$ of the $\mathrm{DFP}_{s}$ (the blue curve) and the $\mathrm{DFP}_{b}$ (the red curve). Note 

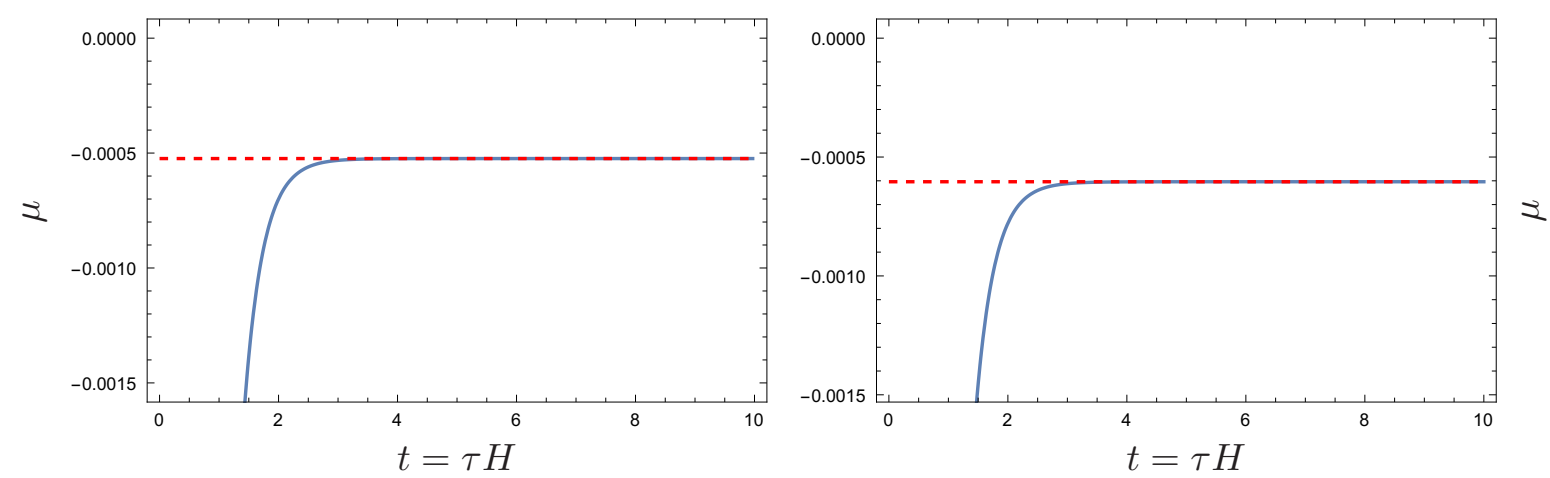

Figure 7. Parameter $\mu(t)$, determining the energy density $\mathcal{E}$, see (A.11), for $\frac{\Lambda}{H}=p_{1}^{<}$(the left panel) and for $\frac{\Lambda}{H}=p_{1}^{>}$(the right panel), see (3.8). The dashed red lines indicate the corresponding $\mathrm{DFP}_{s}$ attractor values.
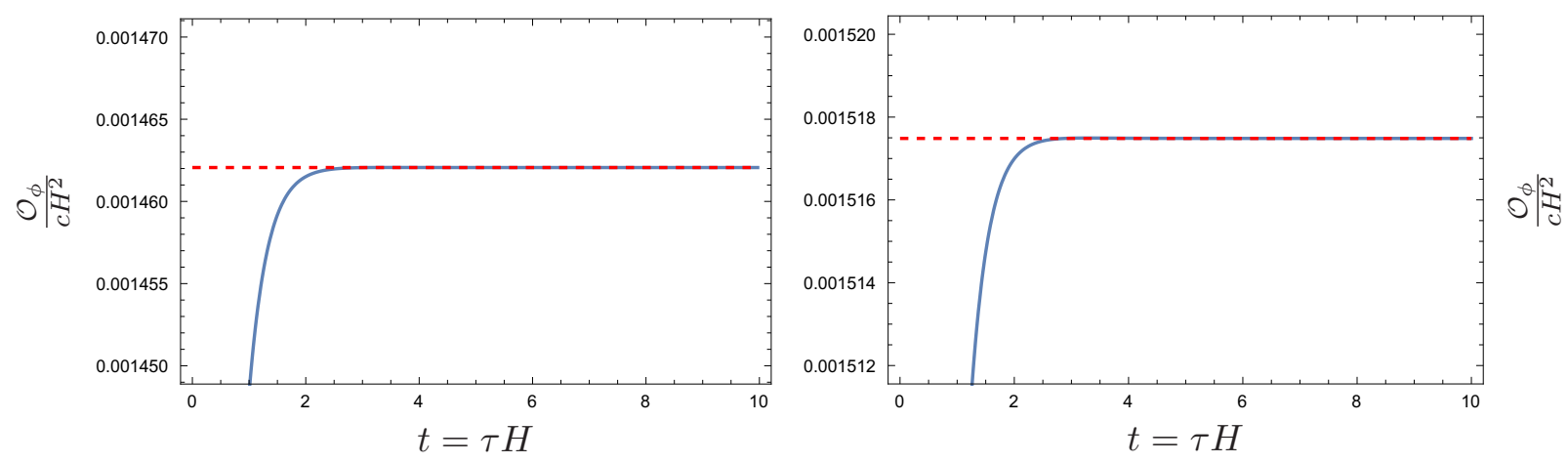

Figure 8. $\mathcal{O}_{\phi}$ expectation values for simulations with $\frac{\Lambda}{H}=p_{1}^{<}$(the left panel) and for $\frac{\Lambda}{H}=p_{1}^{>}$ (the right panel), see (3.8). The dashed red lines indicate the corresponding $\mathrm{DFP}_{s}$ attractor values.

that whenever both exist, the VEE density of the $\mathrm{DFP}_{b}$ is the large one - suggesting that it, rather than the $\mathrm{DFP}_{s}$, is the attractor of the late-time dynamics. However, whenever the $\mathrm{DFP}_{s}$ exists, see (3.6), the symmetry preserving dynamical fixed point ( $\mathrm{DFP}_{s}$ ) is perturbatively stable, see figure 3 . This immediately implies that the $\mathrm{DFP}_{s}$ must be nonperturbatively unstable, if $\frac{\Lambda}{H}$ is in the range (3.6). Our numerical simulations confirm that this is indeed the case.

An interesting twist to the story is the fact that the $\mathrm{DFP}_{b}$ is always perturbatively unstable. The technical details are collected in appendix C.2. In figure 6 we present the spectrum of the unstable QNM in the $\mathrm{DFP}_{b}$ as a function of the order parameter $\mathcal{O}_{\chi}$ (the left panel), and of $\frac{\Lambda}{H}$ (the right panel).

\subsection{Dynamics of the holographic model with linearized $\mathbb{Z}_{2}^{\chi}$ sector}

Technical details used to develop the simulation code are collected in appendix E. The simulations are done with time $\tau$ measured in units of the Hubble time, see (A.4). As a warm-up, we present here the simulation results of our holographic model in the limit when the backreaction of the symmetry breaking sector $\chi$, the $\mathcal{L}_{i}$ Lagrangian of the effective 

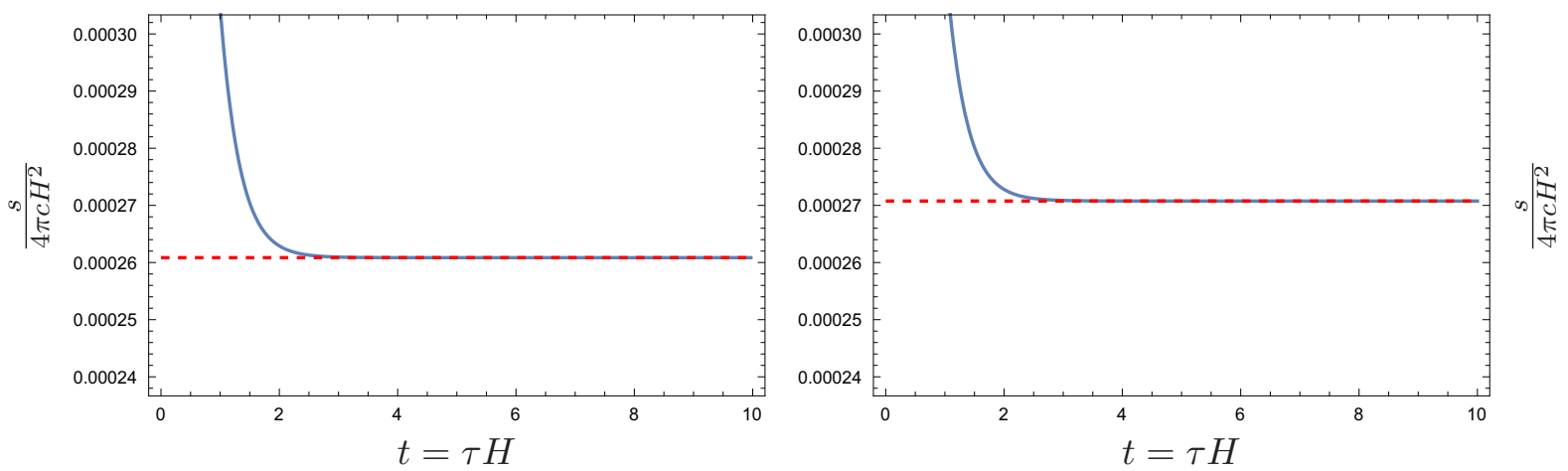

Figure 9. Dynamical entropy density for simulations with $\frac{\Lambda}{H}=p_{1}^{<}$(the left panel) and for $\frac{\Lambda}{H}=p_{1}^{>}$ (the right panel), see (3.8). The dashed red lines indicate the corresponding $\mathrm{DFP}_{s}$ attractor values of the vacuum entanglement entropy density $s_{\text {ent }}$.
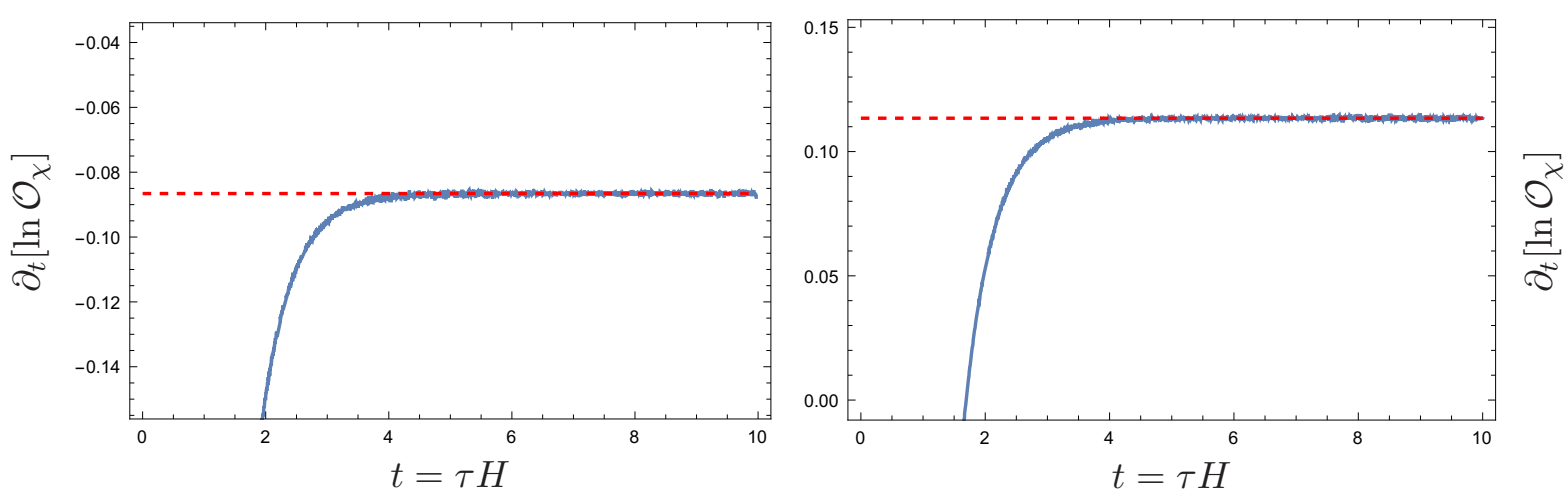

Figure 10. Dynamics of the $\mathbb{Z}_{2}^{\chi}$ symmetry breaking order parameter for simulations with $\frac{\Lambda}{H}=p_{1}^{<}$ (the left panel) and for $\frac{\Lambda}{H}=p_{1}^{>}$(the right panel), see (3.8). The dashed red lines indicate the expected late-time asymptotes, as predicted from the QNM analysis, see (3.9) and (3.10).

action (2.1), is neglected. The remaining $\mathcal{L}_{\mathrm{CFT}}+\mathcal{L}_{r}$ sector is simulated fully nonlinear, ${ }^{8}$ and the $\chi$-sector is evolved as a 'probe'.

We use initial conditions as described in appendix E.3 with

$$
\mathcal{A}_{p}=0.1, \quad \mathcal{A}_{q}=1,\left.\quad \mu\right|_{t=0}=-0.1
$$

Since $\chi$-dynamics is linear and in the probe approximation, the amplitude of the initial profile $\mathcal{A}_{q}$ is an irrelevant overall scale parameter. The initial value of $\mu(t)$ determines the energy density of the initial holographic state, see (A.11). We compare simulation runs for two values of the parameter $p_{1}$ :

$$
p_{1} \equiv \frac{\Lambda}{H}=\left\{p_{1}^{<} \equiv 0.55, p_{1}^{>} \equiv 0.57\right\} .
$$

Note that $p_{1}^{<}<p_{1,4}^{\text {crit }}$, while $p_{1}^{>}>p_{1,4}^{\text {crit }}$ - thus we expect that simulations with $p_{1}=0.55$ would relax to the corresponding $\mathrm{DFP}_{s}$ with

$$
\mathcal{O}_{\chi} \propto e^{-i \hat{\omega}_{4}^{<} \cdot t}, \quad \hat{\omega}_{4}^{<}=-i 0.086582(6),
$$

\footnotetext{
${ }^{8}$ This was done originally in [14].
} 

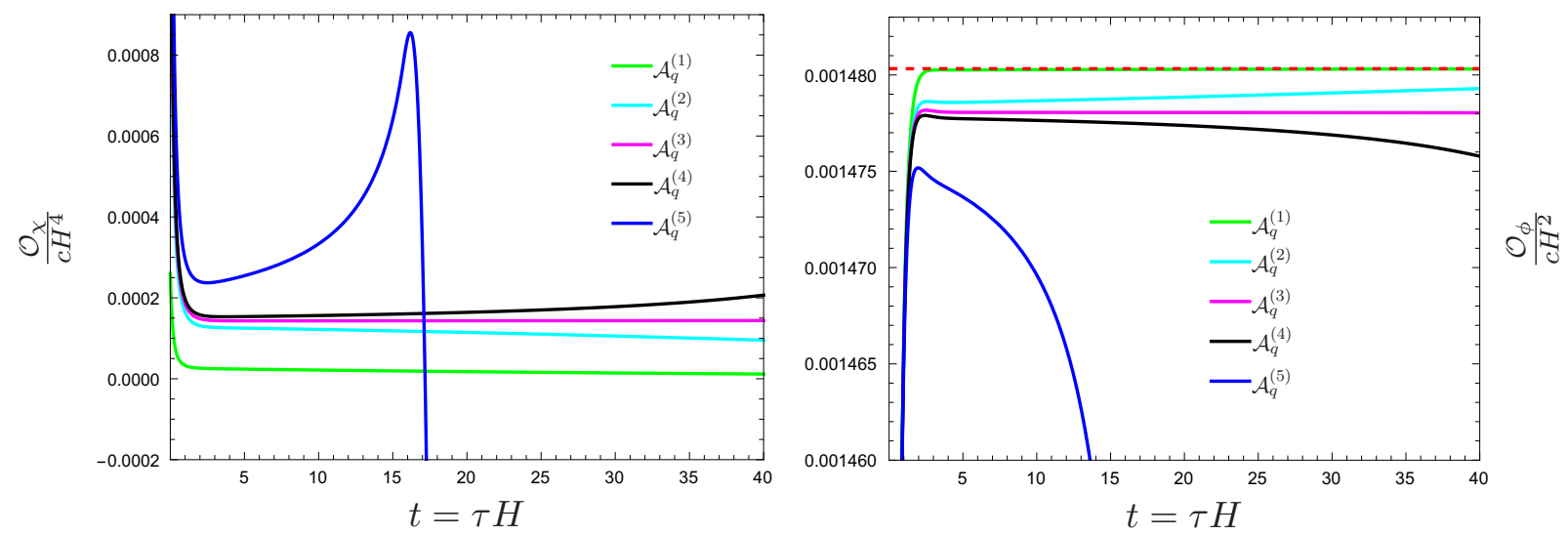

Figure 11. Evolution of expectation values of $\mathcal{O}_{\chi}$ (the left panel) and $\mathcal{O}_{\phi}$ (the right panel) for simulations at $\frac{\Lambda}{H}=p_{1}^{*}(3.13)$, when both the $\mathrm{DFP}_{s}$ and the $\mathrm{DFP}_{b}$ exist. Initial conditions for the amplitude of the $\mathbb{Z}_{2}^{\chi}$ symmetry breaking order parameter $\mathcal{A}_{q}$, see $\left(\right.$ E.23), with $\mathcal{A}_{q}^{(i)}<\mathcal{A}_{q}^{\text {crit }} \approx \mathcal{A}_{q}^{(i)}$, see (3.12), evolve to the $\operatorname{DFP}_{s}$; while those with $\mathcal{A}_{q}^{(i)}>\mathcal{A}_{q}^{\text {crit }}$ eventually evolve to a naked singularity. The fine-tuned $\mathcal{A}_{q}^{(3)}$ initial condition approaches the perturbatively unstable $\mathrm{DFP}_{b}$ at late times.

while those with $p_{1}=0.57$ would highlight the corresponding $\mathrm{DFP}_{s}$ perturbative instability

$$
\mathcal{O}_{\chi} \propto e^{-i \hat{\omega}_{4}^{>} \cdot t}, \quad \hat{\omega}_{4}^{<}=+i 0.113428
$$

for $t \gg 1$. The values $\hat{\omega}_{4}^{<}$and $\hat{\omega}_{4}^{>}$are extracted from the data files used to generate figure 3 .

Figures 7-9 show that the $\mathrm{DFP}_{s}$ is indeed the attractor of the late-time dynamics at $\tau H \gg 1$ in the $\mathbb{Z}_{2}^{\chi}$-symmetric sector, for both of the select values of $p_{1}$ in (3.8). There are no instabilities in this sector, as established in [14]. The solid curves represent the timeseries of the corresponding observables (extracted from the FORTRAN simulation code), and the dashed red lines indicate the attractor values of these observables (extracted from the Wolfram Mathematica codes for DFPs).

Figure 10 presents the evolution of the $\mathbb{Z}_{2}^{\chi}$ symmetry breaking order parameter $\mathcal{O}_{\chi}$ for both of the select values of $p_{1}$ in (3.8), in the probe approximation (the solid curves). The late-time dynamics of the order parameter agrees with the QNM analysis (3.9) and (3.10), represented by the dashed red lines.

\section{4 $\mathrm{DFP}_{s}$ or $\mathrm{DFP}_{b}$ ?}

In this section we present results of the simulations with initial conditions as described in appendix E.3 with

$$
\mathcal{A}_{p}=0.1,\left.\quad \mu\right|_{t=0}=-0.1
$$

and a set of amplitudes $\mathcal{A}_{q}^{(i)}$ of the $\mathbb{Z}_{2}^{\chi}$ symmetry breaking initial conditions:

$$
\mathcal{A}_{q}^{(i)}=\{\underbrace{0.1}_{i=1}, 0.5, \underbrace{0.564}_{i=3}, 0.6, \underbrace{0.9}_{i=5}\} .
$$

We perform simulations at

$$
\frac{\Lambda}{H}=p_{1}^{*}=0.5566<p_{1,4}^{\mathrm{crit}}
$$



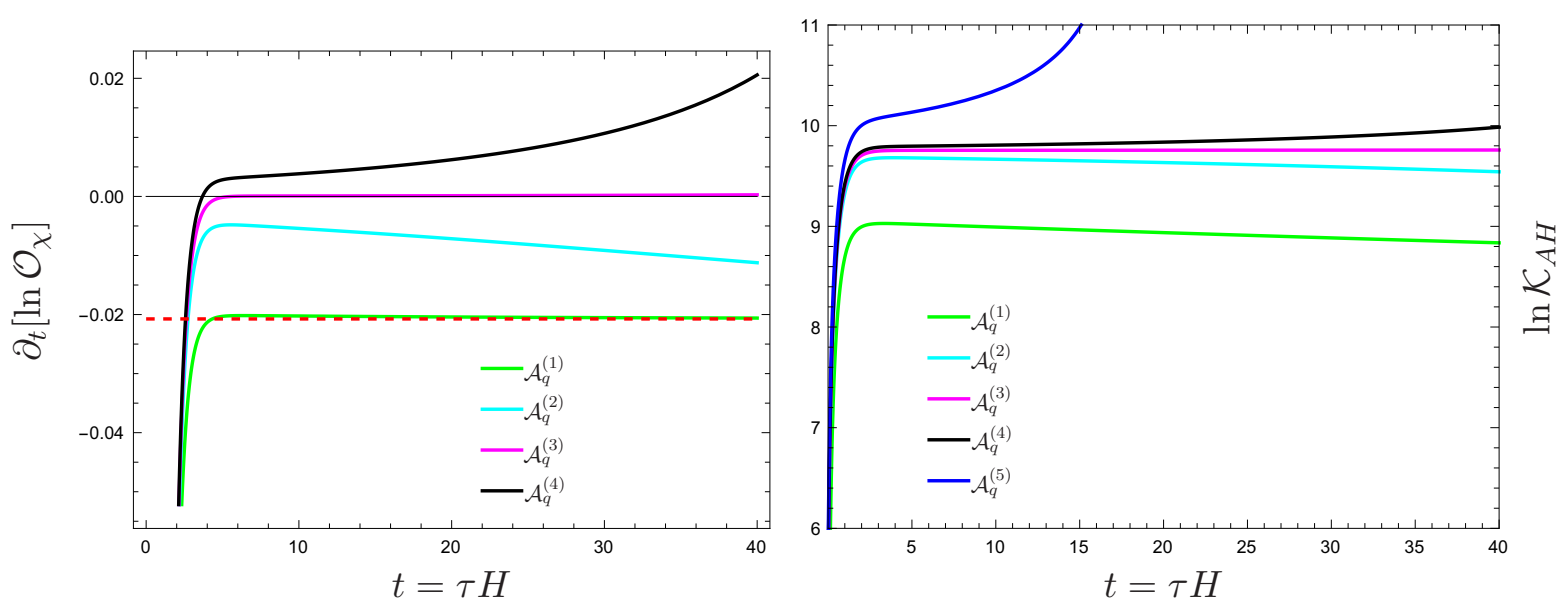

Figure 12. The growth rate of the expectation value of $\mathcal{O}_{\chi}$ (the left panel), and the Kretschmann scalar $\mathcal{K}$ (E.32), evaluated at the apparent horizon (the right panel) for simulations at $\frac{\Lambda}{H}=p_{1}^{*}(3.13)$, when both the $\mathrm{DFP}_{s}$ and the $\mathrm{DFP}_{b}$ exist. The small amplitude initial condition $\mathcal{A}_{q}^{(1)}$ (the green curve) approaches the $\mathrm{DFP}_{s}$ with the appropriate decay rate, determined by the $\mathrm{DFP}_{s} \mathrm{QNM}$ with $\operatorname{Im}\left[\hat{\omega}_{4}\left(p_{1}^{*}\right)\right]$ (the dashed red line). The fine-tuned initial condition $\mathcal{A}_{q}^{(3)}$ (the magenta curve) approaches at late times the $\mathrm{DFP}_{b}$ - it has (close to) vanishing growth rate. Initial configurations with $\mathcal{A}_{q}>\mathcal{A}_{q}^{\text {crit }}$ result in uncontrollable growth of the Kretschmann scalar at the apparent horizon (the black and the blue curves) at late times.

Note that at select value of $p_{1}^{*}$ both the $\mathrm{DFP}_{s}$ and the $\mathrm{DFP}_{b}$ exist:

- the $\mathrm{DFP}_{s}$ is perturbatively stable;

- the $\mathrm{DFP}_{b}$ is perturbatively unstable;

- however,

$$
s_{\mathrm{ent}}\left(\mathrm{DFP}_{b}\right)>s_{\mathrm{ent}}\left(\mathrm{DFP}_{s}\right) .
$$

Given above, we expect that there is a critical amplitude $\mathcal{A}_{q}^{\text {crit }}$, such that initial configurations with $\mathcal{A}_{q}<\mathcal{A}_{q}^{\text {crit }}$ evolve to the $\mathrm{DFP}_{s}$, while configurations with $\mathcal{A}_{q}>\mathcal{A}_{q}^{\text {crit }}$ would evolve to a naked singularity. $\mathcal{A}_{q}>\mathcal{A}_{q}^{\text {crit }}$ initial conditions can not evolve to the $\mathrm{DFP}_{b}$, as it is unstable, neither can they evolve to the $\mathrm{DFP}_{s}$ since a more entropic configuration is available. This is indeed what we find, with

$$
\mathcal{A}_{q}^{\text {crit }} \approx \mathcal{A}_{q}^{(3)}
$$

In figure 11 we present the evolution of $\mathcal{O}_{\chi}$ (the left panel) and $\mathcal{O}_{\phi}$ (the right panel) for select values of $\mathcal{A}_{q}$, see (3.12). For small amplitudes, $\mathcal{A}_{q}^{(1)}$ and $\mathcal{A}_{q}^{(2)}$, the late-time attractor remains the $\mathrm{DFP}_{s}$ - note in particular that for $\mathcal{A}_{q}^{(1)}$ (the green curves), rather quickly, the expectation value of $\mathcal{O}_{\chi}$ decays to zero, restoring $\mathbb{Z}_{2}^{\chi}$ symmetry, while the expectation value of $\mathcal{O}_{\phi}$ approaches the corresponding attractor value of the $\mathrm{DFP}_{s}$ (the dashed red line). Initial configurations $\mathcal{A}_{q}^{(4)}$ and $\mathcal{A}_{q}^{(5)}$ result in uncontrollable growth of the expectation values, more pronounced for $\mathcal{A}_{q}^{(5)}$ (the blue curves). The fine-tuned initial condition with $\mathcal{A}_{q}^{(3)}$ evolves (close) to the $\mathrm{DFP}_{b}$ (the magenta curves). 

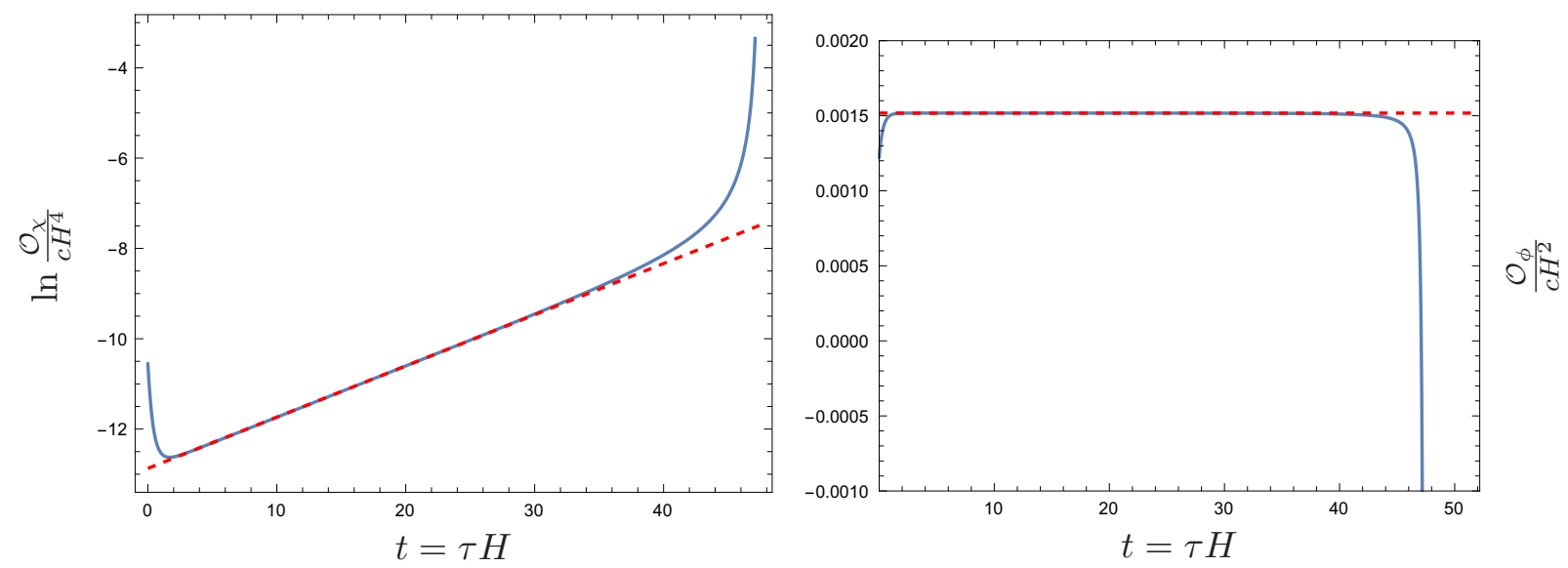

Figure 13. Evolution of expectation values of $\mathcal{O}_{\chi}$ (the left panel) and $\mathcal{O}_{\phi}$ (the right panel) for a simulation at $\frac{\Lambda}{H}=\tilde{p}_{1}(3.17)$, when only the $\mathrm{DFP}_{s}$ exists. Given that the initial amplitude in the $\mathbb{Z}_{2}^{\chi}$ symmetry broken sector is small, see (3.16), its evolution is probe-like on the $\mathrm{DFP}_{s}$ characterized by (3.17). At late times the nonlinearities in the gravitational scalar sector grow without bounds.
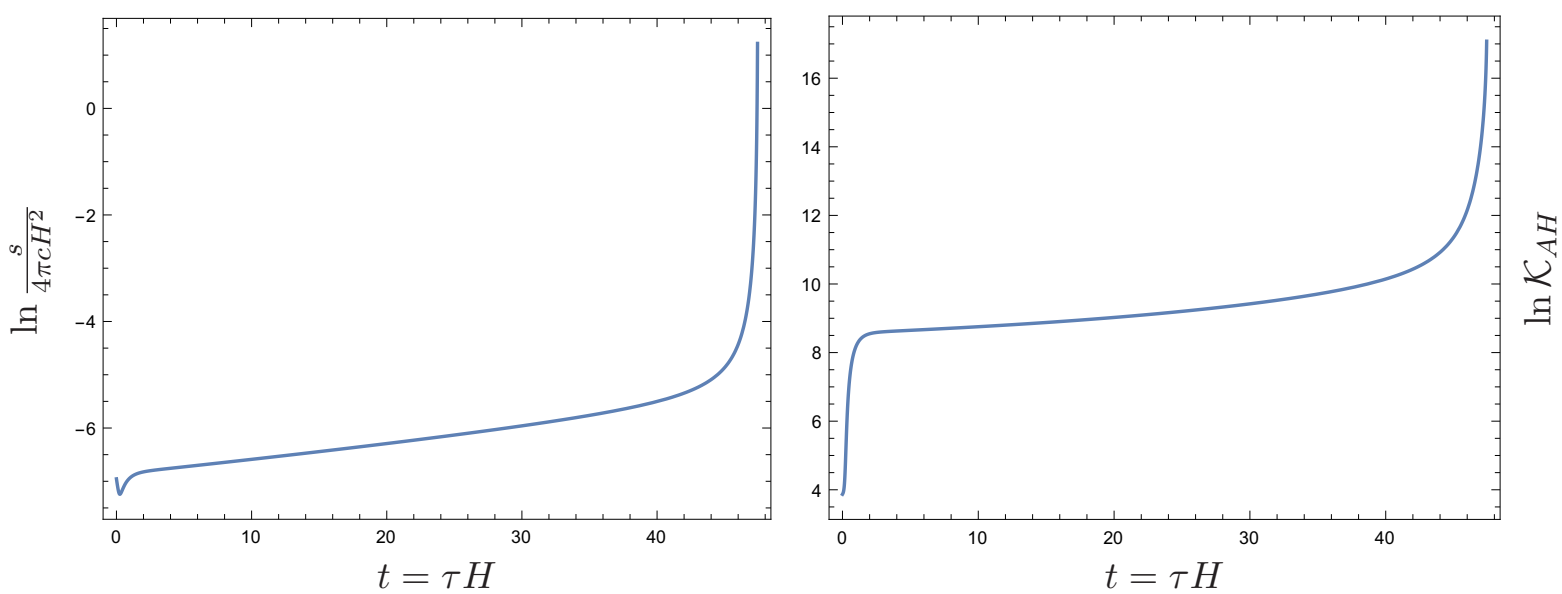

Figure 14. The evolution of the entropy density $s$ (the left panel), and the Kretschmann scalar $\mathcal{K}$ (E.32), evaluated at the apparent horizon (the right panel) for a simulation at $\frac{\Lambda}{H}=\tilde{p}_{1}(3.17)$, when only the $\mathrm{DFP}_{s}$ exists. Since the $\mathrm{DFP}_{s}$ is unstable at (3.17), both quantities grow without bounds at late times.

In figure 12 we extract the growth rate of $\mathcal{O}_{\chi}$ (the left panel), and present the evolution of the Kretschmann scalar $\mathcal{K}$ (E.32), evaluated at the apparent horizon. Initial condition $\mathcal{A}_{q}^{(1)}$ quickly approaches the decay rate set by the corresponding $\mathrm{DFP}_{s}$ (the red dashed line). Since the fine-tuned initial condition $\mathcal{A}_{q}^{(3)}$ evolves to the $\mathrm{DFP}_{b}$, the late-time growth rate of $\mathcal{O}_{\chi}$ is close to zero (the magenta curve). The Kretschmann scalar $\mathcal{K}_{A H}$ grows (apparently unboundedly) at late times for initial conditions with $\mathcal{A}_{q}>\mathcal{A}_{q}^{\text {crit }}$ - the growth is the faster, the larger is the initial amplitude of the $\mathbb{Z}_{2}^{\chi}$ symmetry breaking. 


\subsection{DFP does not always exist}

Dynamics described in the previous section is an example when a DFP is absent for certain initial states (those with $\mathcal{A}_{q}>\mathcal{A}_{q}^{\text {crit }}$ ), but is an attractor for some other initial configurations (those with $\mathcal{A}_{q}<\mathcal{A}_{q}^{\text {crit }}$ ). In this section we present results of the simulations with initial conditions as described in appendix E.3 with

$$
\mathcal{A}_{p}=0.1, \quad \mathcal{A}_{q}=0.01,\left.\quad \mu\right|_{t=0}=-0.1 .
$$

We perform simulations at

$$
\frac{\Lambda}{H}=\tilde{p}_{1}=0.57>p_{1,4}^{\text {crit }} .
$$

Note that at select value of $\tilde{p}_{1}$ only the $\mathrm{DFP}_{s}$ exists, however, it is perturbatively unstable to $\mathbb{Z}_{2}^{\chi}$ symmetry breaking fluctuations. Thus we expect that, no matter how small $\mathcal{A}_{q}$ is, the configuration would evolve to a naked singularity, i.e., this is an example when a DFP does not exist, no matter the initial state.

In figure 13 we present the evolution of $\mathcal{O}_{\chi}$ (the left panel) and $\mathcal{O}_{\phi}$ (the right panel). Note that the benefit of the small amplitude $\mathcal{A}_{q}$ in (3.16) is that the $\mathbb{Z}_{2}^{\chi}$-symmetric sector of the model approaches the $\operatorname{DFP}_{s}$ (within several Hubble times $t \sim 1$ ), while the $\mathbb{Z}_{2}^{\chi}$ symmetry breaking sector effectively evolves as a probe till $t \sim 35$. For yet larger values of $t$, the nonlinear effects drive the evolution of the geometry to a naked singularity. The red dashed line in the left panel presents the prediction for the growth of $\mathcal{O}_{\chi}$ on top of $\mathrm{DFP}_{s}$ specified by (3.17), driven by the QNM with $\hat{\omega}_{4}\left(\tilde{p}_{1}\right)$. The red dashed line in the right panel presents the expectation value of $\mathcal{O}_{\phi}$ in $\mathrm{DFP}_{s}$ specified by (3.17).

In figure 14 we confirm that the instability of the $\mathrm{DFP}_{s}$, and the absence of any other DFP at $\frac{\Lambda}{H}=\tilde{p}_{1}$, results in unbounded growth of the entropy density $s$ (the left panel) and the Kretschmann scalar evaluated at the apparent horizon $\mathcal{K}_{A H}$ at late times.

\subsection{Dynamics of $\mathrm{DFP}_{b}$}

In this section we present results of the simulation when the initial condition is, to a very good approximation, the $\mathrm{DFP}_{b}$ - see appendix E.4 for the technical details. We choose the $\mathrm{DFP}_{b}$ at (3.13). Since the $\mathrm{DFP}_{b}$ is perturbatively unstable, the numerical noise introduced transcribing the input from Wolfram Mathematica to the simulation code triggers the instability. The growth rate of the initial noise is controlled by the unstable QNM of the $\mathrm{DFP}_{b}$ with $\operatorname{Im}\left[\hat{\omega}\left(p_{1}^{*}\right)\right]$. This is indeed what we find: in figures 15 and 16 we present the evolution of $\mathcal{O}_{\phi}$ and $\mathcal{O}_{\chi}$ (the solid curves). The dashed red lines indicate predictions of the corresponding values from the $\mathrm{DFP}_{b}$ at (3.13).

\section{Conclusions}

In this paper we proposed a formal concept of a dynamical fixed point (DFP) of a macroscopic system: the main distinction from the thermal equilibrium is the condition that the entropy current divergence of the system at late times is strictly positive. The clear shortcoming (with respect to practical applications) is the necessity of the precise definition of 

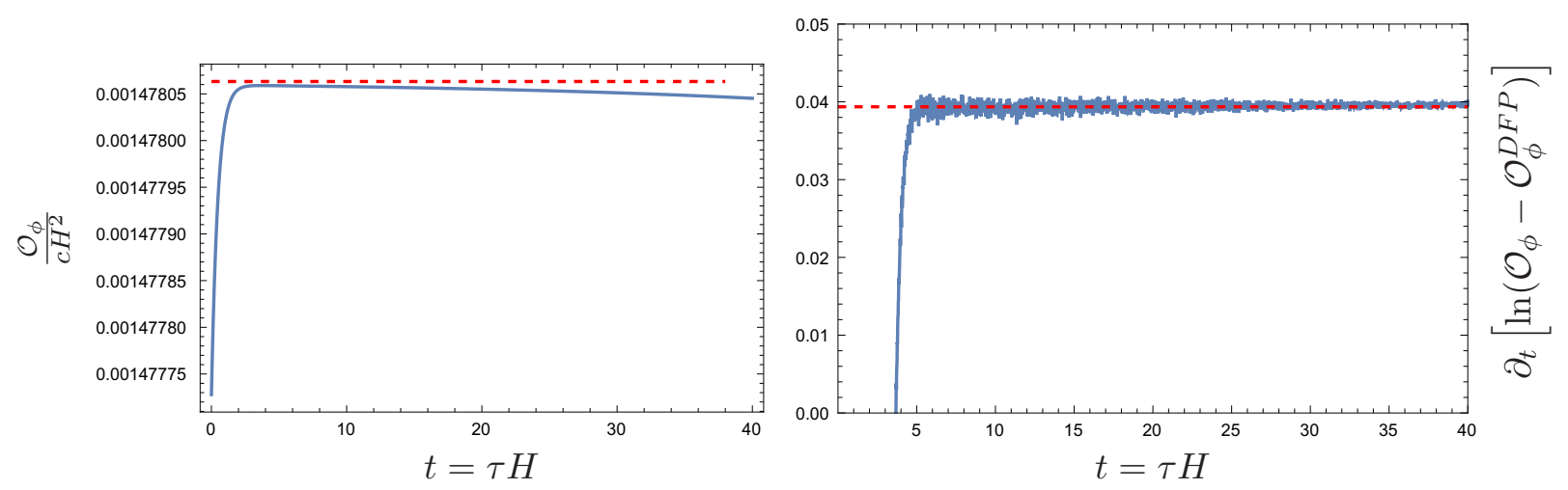

Figure 15. Evolution of $\mathcal{O}_{\phi}$ with the initial configuration set as the $\mathrm{DFP}_{b}$ at $\frac{\Lambda}{H}=p_{1}^{*}$, see (3.13). The instability of the $\mathrm{DFP}_{b}$ causes the noise introduced by the initial conditions to grow at the rate predicted by the unstable quasinormal mode of the $\mathrm{DFP}_{b}$ as $\operatorname{Im}\left[\hat{\omega}\left(p_{1}^{*}\right)\right]$ (the dashed red line in the right panel).
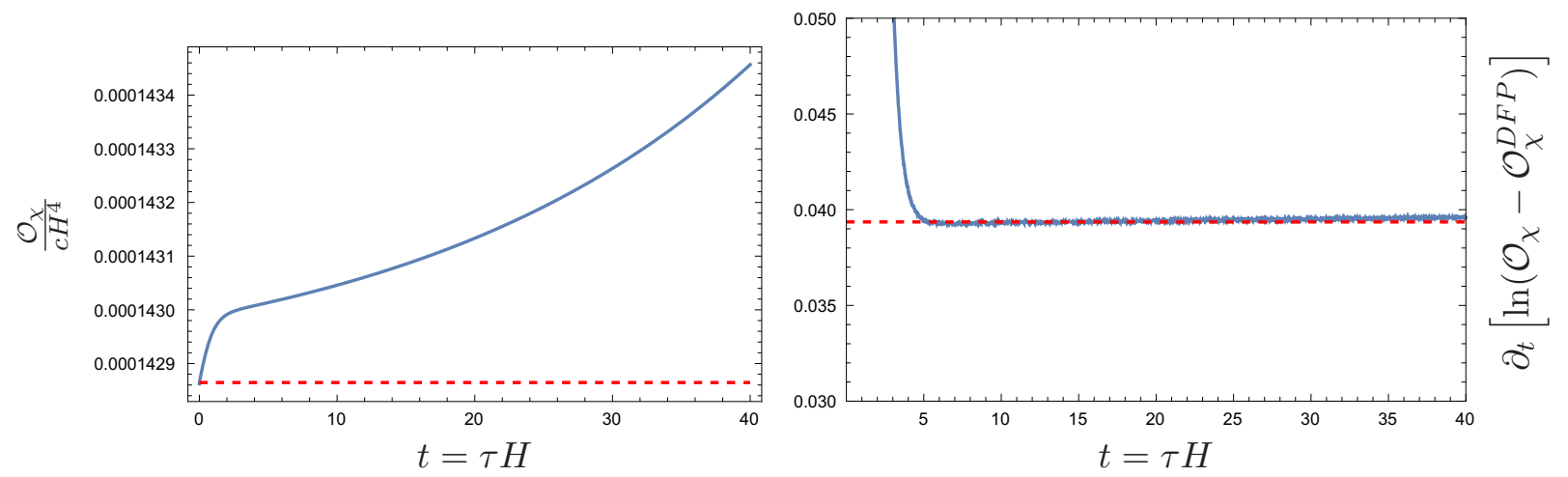

Figure 16. Evolution of $\mathcal{O}_{\chi}$ with the initial configuration set as the $\mathrm{DFP}_{b}$ at $\frac{\Lambda}{H}=p_{1}^{*}$, see (3.13). The instability of the $\mathrm{DFP}_{b}$ causes the noise introduced by the initial conditions to grow at the rate predicted by the unstable quasinormal mode of the $\mathrm{DFP}_{b}$ as $\operatorname{Im}\left[\hat{\omega}\left(p_{1}^{*}\right)\right]$ (the dashed red line in the right panel).

the entropy current of a system far-from-equilibrium. In strongly coupled systems, which have a dual holographic description, the candidate entropy current can be constructed from the gravitational entropy density of the apparent horizon.

There are multiple avenues for future studies:

- regarding the 'big questions',

- We studied DFPs of a holographic system driven by the background space-time expansion. Can one define interesting protocols for time-dependence of coupling constants, masses, external magnetic fields of a holographic model, which result in DFPs?

- What are the interesting plasma hydrodynamic flows that do not have thermal equilibrium as a late-time attractor?

- How universal are DFPs, and is there a universal replacement for hydrodynamics, as a theory describing the approach to a DFP? 
- Can DFPs be studied for theories at weak coupling? Are there phenomenological applications to cosmology?

- Related to above, can examples of DFPs be constructed within kinetic theory? In [22] $\mathcal{N}=2^{*}$ gauge theory de Sitter DFPs were constructed at strong coupling: they are characterized by the expectation values of certain dimension $\Delta=2$ and $\Delta=3$ gauge invariant operators. It was shown that these expectation values are not the thermal expectation values of the theory in Minkowski space-time at temperature set equal to de Sitter 'temperature' $\frac{H}{2 \pi}$ (also at strong coupling). The question whether weaklycoupled DFPs (if they exist) of quantum field theories in de Sitter can be interpreted as thermal states of pair-produced particles at de Sitter temperature $T=\frac{H}{2 \pi}$ is open.

- regarding the holographic toy model discussed here, and its generalizations,

- What are the properties of the $\mathrm{DFP}_{b}$, triggered by the instabilities of the QNMs with $\hat{\omega}_{n}$ for $n>4$ ?

- What is the landscape of DFPs of the model defined on compact spatial manifolds?

- What is the role of supersymmetry and the unboundedness of the scalar potentials in the dual gravitation effective actions ${ }^{9}$ on DFPs?

- Analysis of the cascading gauge theory in de Sitter space-time [12] is the analysis of its DFPs. Whether these DFPs are stable or not, and when are they the attractors of the late-time evolution remains to be analyzed.

\section{Acknowledgments}

This research is supported in part by Perimeter Institute for Theoretical Physics. Research at Perimeter Institute is supported in part by the Government of Canada through the Department of Innovation, Science and Economic Development Canada and by the Province of Ontario through the Ministry of Colleges and Universities. This work was further supported by NSERC through the Discovery Grants program.

\section{A Holographic EOMs, the boundary asymptotics and the renormaliza- tion}

Einstein equations from (2.1) define the following evolution equations of motion:

$$
\begin{aligned}
0 & =\left(d_{+} \Sigma\right)^{\prime}+d_{+} \Sigma(\ln \Sigma)^{\prime}-\frac{3}{2} \Sigma-\frac{1}{4} \Sigma\left(\phi^{2}-2 \chi^{2}-g \phi^{2} \chi^{2}\right), \\
0 & =\left(d_{+} \phi\right)^{\prime}+d_{+} \phi(\ln \Sigma)^{\prime}+\frac{d_{+} \Sigma}{\Sigma} \phi^{\prime}+\phi\left(1-g \chi^{2}\right), \\
0 & =\left(d_{+} \chi\right)^{\prime}+d_{+} \chi(\ln \Sigma)^{\prime}+\frac{d_{+} \Sigma}{\Sigma} \chi^{\prime}-\chi\left(2+g \phi^{2}\right), \\
0 & =A^{\prime \prime}-2 \frac{d_{+} \Sigma}{\Sigma^{2}} \Sigma^{\prime}+\frac{1}{2} d_{+} \phi \phi^{\prime}+\frac{1}{2} d_{+} \chi \chi^{\prime},
\end{aligned}
$$

\footnotetext{
${ }^{9}$ The work [23] could be a useful resource.
} 
together with the Hamiltonian constraint equation:

$$
0=\Sigma^{\prime \prime}+\frac{1}{4} \Sigma\left(\left(\phi^{\prime}\right)^{2}+\left(\chi^{\prime}\right)^{2}\right)
$$

and the momentum constraint equation:

$$
\begin{aligned}
0= & d_{+}^{2} \Sigma-2 A\left(d_{+} \Sigma\right)^{\prime}-\frac{d_{+} \Sigma}{\Sigma^{2}}\left(A \Sigma^{2}\right)^{\prime} \\
& +\frac{1}{4} \Sigma\left(\left(d_{+} \phi\right)^{2}+\left(d_{+} \chi\right)^{2}+2 A\left(6+\phi^{2}-2 \chi^{2}-g \phi^{2} \chi^{2}\right)\right),
\end{aligned}
$$

where $^{\prime} \equiv \partial_{r}$ and $d_{+} \equiv \partial_{\tau}+A \partial_{r}$. The constraint equations are preserved by the evolution equations provided they are satisfied at a given time-like surface - which in our case is the AdS boundary.

It is convenient to compactify the gravitational dual spatial domain, introducing a new dimensionless radial coordinate $x$, and measuring time in Hubble units $t$ :

$$
x \equiv \frac{H}{r}, \quad t \equiv H \tau .
$$

Further introducing

$$
\Sigma(t, x) \equiv H \exp (t) \sigma(t, x), \quad A(t, x) \equiv H^{2} a(t, x),
$$

the $H$ dependence drops out of (A.1) and (A.3):

$$
\begin{aligned}
& 0=\left(d_{+} \sigma\right)^{\prime}+d_{+} \sigma(\ln \sigma)^{\prime}+2 \sigma^{\prime}+\frac{3 \sigma}{2 x^{2}}+\frac{\sigma}{4 x^{2}}\left(\phi^{2}-2 \chi^{2}-g \phi^{2} \chi^{2}\right), \\
& 0=\left(d_{+} \phi\right)^{\prime}+d_{+} \phi(\ln \sigma)^{\prime}+\left(\frac{d_{+} \sigma}{\sigma}+1\right) \phi^{\prime}-\frac{\phi}{x^{2}}\left(1-g \chi^{2}\right), \\
& 0=\left(d_{+} \chi\right)^{\prime}+d_{+} \chi(\ln \sigma)^{\prime}+\left(\frac{d_{+} \sigma}{\sigma}+1\right) \chi^{\prime}+\frac{\chi}{x^{2}}\left(2+g \phi^{2}\right), \\
& 0=a^{\prime \prime}+\frac{2}{x} a^{\prime}+\frac{2 \sigma^{\prime}}{x^{2} \sigma}\left(\frac{d_{+} \sigma}{\sigma}+1\right)-\frac{\phi^{\prime}}{2 x^{2}} d_{+} \phi-\frac{\chi^{\prime}}{2 x^{2}} d_{+} \chi,
\end{aligned}
$$

and

$$
\begin{aligned}
0= & \sigma^{\prime \prime}+\frac{2}{x} \sigma^{\prime}+\frac{1}{4} \sigma\left(\left(\phi^{\prime}\right)^{2}+\left(\chi^{\prime}\right)^{2}\right), \\
0= & d_{+}^{2} \sigma+2 x^{2} a\left(d_{+} \sigma\right)^{\prime}+d_{+} \sigma\left(\frac{x^{2}}{\sigma^{2}}\left(a \sigma^{2}\right)^{\prime}+2\right)+\frac{x^{2}}{\sigma^{3}}\left(a \sigma^{4}\right)^{\prime} \\
& +\frac{1}{4} \sigma\left(\left(d_{+} \phi\right)^{2}+\left(d_{+} \chi\right)^{2}+2 a\left(6+\phi^{2}-2 \chi^{2}-g \phi^{2} \chi^{2}\right)+4\right),
\end{aligned}
$$

where now ${ }^{\prime} \equiv \partial_{x}$ and $d_{+} \equiv \partial_{\tau}-a x^{2} \partial_{x}$. 
The general asymptotic boundary $(x \rightarrow 0)$ solution of the equations of motion, given by

$$
\begin{aligned}
\sigma= & \frac{1}{x}+\lambda(t)-\frac{1}{8} p_{1}^{2} x+\mathcal{O}\left(x^{2}\right), \\
a= & \frac{1}{2 x^{2}}+\frac{\lambda(t)-1}{x}-\frac{1}{8} p_{1}^{2}+\frac{1}{2} \lambda(t)^{2}-\lambda-\dot{\lambda}(t) \\
& +\left(\mu(t)-\frac{1}{4} p_{1} p_{2}(t)-\frac{1}{4} p_{1}^{2} \lambda(t)+\frac{1}{6} p_{1}^{2}\right) x+\mathcal{O}\left(x^{2}\right), \\
\phi= & p_{1} x+p_{2}(t) x^{2}+\mathcal{O}\left(x^{3}\right) \\
\chi= & q_{4}(t) x^{4}+\mathcal{O}\left(x^{5}\right)
\end{aligned}
$$

with ${ }^{\cdot} \equiv \partial_{t}$, is characterized by a single constant $p_{1}$, and four dynamical variables $\left\{p_{2}(t), q_{4}(t), \lambda(t), \mu(t)\right\}$.

Notice that the first equation in (A.7) can be integrated to yield

$$
\sigma^{\prime}(t, x)=-\frac{1}{x^{2}}-\frac{1}{4 x^{2}} \int_{0}^{x} d s\left\{s^{2} \sigma(t, s)\left(\left(\phi^{\prime}(t, s)\right)^{2}+\left(\chi^{\prime}(t, s)\right)^{2}\right)\right\}
$$

where we used the asymptotes (A.8), implying that, $\sigma(t, x)$ is necessarily positive,

$$
\sigma^{\prime}(t, x)<0
$$

for all values of $x$. This fact will be important for the dynamical entropy growth theorem, see appendix D.

The observables of interest, i.e., the energy density $\mathcal{E}(t)$, the pressure $P(t)$, and the expectation values of the operators $\mathcal{O}_{\phi}(t)$ and $\mathcal{O}_{\chi}(t)$ (dual to the bulk scalars $\phi$ and $\chi$ correspondingly) can be computed following the holographic renormalization of the model [14]:

$$
\begin{aligned}
\frac{2 \kappa^{2}}{H^{3}} \mathcal{E}(t) & =-4 \mu(t)+\frac{1}{3} p_{1}^{2}+\left(\delta_{1} p_{1}^{3}+2 \delta_{2} p_{1}\right), \\
\frac{2 \kappa^{2}}{H^{3}} P(t) & =-2 \mu(t)-\frac{1}{3} p_{1}^{2}+\frac{1}{2} p_{1}\left(p_{2}(t)+\lambda(t) p_{1}\right)+\left(-\delta_{1} p_{1}^{3}-2 \delta_{2} p_{1}\right), \\
\frac{2 \kappa^{2}}{H^{2}} \mathcal{O}_{\phi}(t) & =-p_{2}(t)-\lambda(t) p_{1}+p_{1}+\left(3 \delta_{1} p_{1}^{2}+6 \delta_{2}\right), \\
\frac{2 \kappa^{2}}{H^{4}} \mathcal{O}_{\chi}(t) & =q_{4}(t),
\end{aligned}
$$

where the terms in brackets, depending on arbitrary constants $\left\{\delta_{1}, \delta_{2}\right\}$, encode the renormalization scheme ambiguities. Independent of the renormalization scheme, these expectation values satisfy the expected conformal Ward identity

$$
-\mathcal{E}+2 P=-p_{1} H \mathcal{O}_{\phi}
$$

Furthermore, the conservation of the stress-energy tensor

$$
\frac{d \mathcal{E}}{d t}+2(\mathcal{E}+P)=0
$$


is a consequence of the momentum constraint (A.3):

$$
0=\dot{\mu}+3 \mu-\frac{p_{1}}{4}\left(p_{2}+\lambda p_{1}\right) .
$$

From now on we choose a scheme with $\delta_{i}=0$.

The remaining parameters $\left\{p_{1}, \lambda(t)\right\}$ have the following interpretation:

- $p_{1}$ is identified with the deformation mass scale $\Lambda(2.6)$,

$$
p_{1}=\frac{\Lambda}{H},
$$

- $\lambda(t)$ is the residual radial coordinate diffeomorphism parameter [19],

$$
\frac{r}{H} \equiv \frac{1}{x} \rightarrow \frac{1}{x}+\lambda(t),
$$

which can be adjusted to keep the apparent horizon at a fixed location, which in our case will be $r=r_{A H}=H$, equivalently, $x_{A H}=1$ :

$$
\left.\left.\left(\partial_{\tau}+A(\tau, r) \partial_{r}\right) \Sigma(t, r)\right|_{r_{A H}} \equiv H^{2} e^{t}\left(d_{+} \sigma(t, x)+\sigma(t, x)\right)\right|_{x_{A H}}=0
$$

To initialize evolution at $t=0$, we provide the bulk scalar profiles,

$$
\phi(t=0, x)=p_{1} x+\mathcal{O}\left(x^{2}\right), \quad \chi(t=0, x)=\mathcal{O}\left(x^{4}\right),
$$

along with the values of $\left\{p_{1}, \mu(t=0)\right\}$, specifying the dual $Q F T_{3}$ mass scale $\Lambda$ (A.18) and the initial state energy density $\mathcal{E}(t=0)(\mathrm{A} .11)$. The Hamiltonian constraint equation in (A.7) is then used to determine an initial profile $\sigma(t=0, x)$. Eqs. (A.6) are then employed to evolve such data (A.21) in time. The second constraint in (A.7), representing the conservation of the boundary stress-energy tensor, is enforced requiring that a dynamical variable $\mu(t)$ in the asymptotic expansion of $a$, see (A.8), evolves following (A.17).

Details of the numerical implementation, specific choices of the initial conditions (A.21) used, and code convergence tests can be found in appendix E.

\section{B $\mathbb{Z}_{2}$-symmetric DFP and its fluctuations}

\section{B.1 $\mathbb{Z}_{2}^{\chi}$-symmetric DFP}

$\mathbb{Z}_{2}^{\chi}$-symmetric dynamical fixed point is the $t \rightarrow \infty$ (late-time) solution of (A.6)-(A.7) with $\chi(t, x) \equiv 0$. Introducing

$$
\lim _{t \rightarrow \infty}\{\sigma, a, \phi\}(t, x)=\left\{\frac{\mathcal{F}(x)}{x}, \frac{\mathcal{G}(x)}{2 x^{2}}, p(x)\right\},
$$

we find from (A.6)-(A.7)

$$
\begin{aligned}
0=\mathcal{F}^{\prime \prime}+\frac{1}{4} \mathcal{F}\left(p^{\prime}\right)^{2} \\
\begin{aligned}
0=p^{\prime \prime}-\frac{2 \mathcal{F} x^{2}}{K}\left(p^{\prime}\right)^{3}+\frac{\mathcal{F} p}{K}\left(p^{\prime}\right)^{2}+\frac{1}{K}( & -\frac{24 x^{2}\left(\mathcal{F}^{\prime}\right)^{2}}{\mathcal{F}}-\frac{2\left(2 \mathcal{F}^{\prime} x-\mathcal{F}\right) p^{2}}{x}+24(x-1) \mathcal{F}^{\prime} \\
& \left.+\frac{12 \mathcal{F}}{x}\right) p^{\prime}-\frac{12 p}{\mathcal{F} x^{2} K}\left(\mathcal{F}^{\prime} x-\mathcal{F}\right)^{2},
\end{aligned}
\end{aligned}
$$


where

$$
K=-\mathcal{F} p^{2}-12 \mathcal{F}^{\prime} x^{2}+6 \mathcal{F}(2 x-1),
$$

along with the algebraic equation for $\mathcal{G}$ :

$$
\mathcal{G}=\frac{2 \mathcal{F} K}{\mathcal{F}^{2} x^{2}\left(p^{\prime}\right)^{2}-12\left(\mathcal{F}^{\prime} x-\mathcal{F}\right)^{2}} .
$$

The late-time apparent horizon is located at $x_{A H}$, such that, see (A.20),

$$
0=-\frac{\mathcal{G}}{2} \frac{d \sigma}{d x}+\left.\sigma\right|_{x_{A H}}
$$

Since $\sigma^{\prime}<0$ (A.10), it is clear from (B.6) that $\mathcal{G}\left(x_{A H}\right)<0$. However, as $\mathcal{G}(x \rightarrow 0)=1$, see (A.8), this implies that $\mathcal{G}$ vanishes at certain $x_{s} \in\left(0, x_{A H}\right)$. Vanishing of $\mathcal{G}$ arises from vanishing of $K$ (B.4), $K\left(x_{s}\right)=0$, making the bulk scalar equation (B.3) 'singular' at this point - note the factor $K$ in the denominator of some terms. This 'singularity' is spurious, and solutions $\{\mathcal{F}, p, \mathcal{G}\}$ are in fact smooth for $x \in\left[0, x_{A H}\right]$. To solve for a dynamical fixed point (B.2)-(B.5), it is convenient to use the residual diffeomorphism parameter $\lambda$ so that $x_{s}$, rather than $x_{A H}$, is kept fixed (as one varies $p_{1}$ ):

$$
x_{s}=\frac{1}{3} .
$$

Thus, we seek solutions of (B.2)-(B.5) subject to the following boundary conditions:

- in the $\mathrm{UV},{ }^{10}$ i.e., as $x \rightarrow 0_{+}$,

$$
\begin{aligned}
\mathcal{F}= & 1+\lambda x-\frac{1}{8} p_{1}^{2} x^{2}+\left(-\frac{1}{24} \lambda p_{1}^{2}-\frac{1}{6} p_{1} p_{2}\right) x^{3}+\mathcal{O}\left(x^{4}\right) \\
p= & p_{1} x+p_{2} x^{2}+\left(\frac{1}{4} p_{1}^{3}-2 \lambda p_{2}+2 p_{2}+2 \lambda p_{1}-\lambda^{2} p_{1}\right) x^{3}+\mathcal{O}\left(x^{4}\right) \\
\mathcal{G}= & 1+(-2+2 \lambda) x+\left(-\frac{1}{4} p_{1}^{2}+\lambda^{2}-2 \lambda\right) x^{2}+\left(-\frac{1}{3} \lambda p_{1}^{2}+\frac{1}{3} p_{1}^{2}-\frac{1}{3} p_{1} p_{2}\right) x^{3} \\
& +\left(-\frac{1}{24} p_{1}^{4}-\frac{1}{6} p_{2}^{2}-\frac{1}{3} \lambda p_{1}^{2}+\frac{1}{6} \lambda^{2} p_{1}^{2}\right) x^{4}+\mathcal{O}\left(x^{5}\right)
\end{aligned}
$$

- in the IR, i.e., , as $y \equiv \frac{1}{3}-x \rightarrow 0_{+}$,

$$
\begin{aligned}
\mathcal{F} & =f_{0}^{s}+\frac{3}{4} f_{0}^{s}\left(\left(p_{0}^{s}\right)^{2}+2\right) y+\mathcal{O}\left(y^{2}\right), \\
p & =p_{0}^{s}-\frac{9}{2} p_{0}^{s} y+\mathcal{O}\left(y^{2}\right), \\
\mathcal{G} & =2 y+\left(3+\frac{3}{2}\left(p_{0}^{s}\right)^{2}\right) y^{2}+\mathcal{O}\left(y^{3}\right) .
\end{aligned}
$$

For a fixed $p_{1}$, alternatively the mass scale $\Lambda$ (A.18), the DFP gravitational dual is characterized by $\left\{p_{2}, \lambda, f_{0}^{s}, p_{0}^{s}\right\}$ - precisely the number of parameters needed to solve the pair

\footnotetext{
${ }^{10}$ Compare with (A.8).
} 
of the second order ODEs (B.2) and (B.3). Notice that $\mu$ is not an independent parameter - from (A.17) at the DFP $\dot{\mu}=0$ leading to

$$
\mu=\frac{p_{1}}{12}\left(p_{2}+\lambda p_{1}\right) .
$$

The solutions for $x \in\left[0, \frac{1}{3}\right)$ are then extended for $x \in\left(\frac{1}{3}, x_{A H}\right]$, solving (B.2) and (B.3), with initial conditions (see (B.9))

$$
\begin{aligned}
\mathcal{F} & =f_{0}^{s}+\frac{3}{4} f_{0}^{s}\left(\left(p_{0}^{s}\right)^{2}+2\right)\left(\frac{1}{3}-x\right)+\mathcal{O}\left(\left(\frac{1}{3}-x\right)^{2}\right), \\
p & =p_{0}^{s}-\frac{9}{2} p_{0}^{s}\left(\frac{1}{3}-x\right)+\mathcal{O}\left(\left(\frac{1}{3}-x\right)^{2}\right), \\
\mathcal{G} & =2\left(\frac{1}{3}-x\right)+\left(3+\frac{3}{2}\left(p_{0}^{s}\right)^{2}\right)\left(\frac{1}{3}-x\right)^{2}+\mathcal{O}\left(\left(\frac{1}{3}-x\right)^{3}\right),
\end{aligned}
$$

while monitoring the AH locator (B.6).

For a finite $p_{1}$, the data sets $\left\{p_{2}, \lambda, f_{0}^{s}, p_{0}^{s}\right\}$ are found numerically, using the shooting method developed in [24]. As originally presented in [14], equations (B.2) and (B.3) can be solved perturbatively in $p_{1}$ :

$$
\begin{aligned}
\mathcal{F}= & 1-x+\frac{x(4 x-1)}{24(x-1)} p_{1}^{2}+\frac{x(4 x-1)\left(23 x^{2}-5 x-5\right)}{3456(x-1)^{3}} p_{1}^{4}+\frac{x}{6220800(x-1)^{5}}\left(49618 x^{5}\right. \\
& \left.-46133 x^{4}+9055 x^{3}-2745 x^{2}+3225 x-645\right) p_{1}^{6}+\frac{x}{87787929600(x-1)^{7}} \\
& \times\left(239535208 x^{7}-287767231 x^{6}+66948945 x^{5}+34436913 x^{4}-23724575 x^{3}\right. \\
& \left.+13512065 x^{2}-5761175 x+823025\right) p_{1}^{8}+\mathcal{O}\left(p_{1}^{10}\right), \\
p= & \frac{x}{(1-x)} p_{1}-\frac{x^{2}(2 x-1)}{9(x-1)^{3}} p_{1}^{3}-\frac{x^{2}\left(875 x^{3}-647 x^{2}+9 x+51\right)}{12960(x-1)^{5}} p_{1}^{5} \\
& -\frac{x^{2}\left(822367 x^{5}-874077 x^{4}+196890 x^{3}+19590 x^{2}+17295 x-9265\right)}{32659200(x-1)^{7}} p_{1}^{7} \\
& +\mathcal{O}\left(p_{1}^{9}\right), \\
\mathcal{G}= & (1-3 x)\left(1-x+\frac{(3 x-1) x}{12(x-1)} p_{1}^{2}+\frac{(3 x-1)\left(19 x^{2}-2 x-5\right) x}{1728(x-1)^{3}} p_{1}^{4}+\frac{x(3 x-1)}{622080(x-1)^{5}}\right. \\
& \times\left(1937 x^{4}-1196 x^{3}+54 x^{2}-204 x+129\right) p_{1}^{6}+\frac{(3 x-1) x}{6270566400(x-1)^{7}}\left(6281809 x^{6}\right. \\
& \left.\left.-5336034 x^{5}-314745 x^{4}+1210180 x^{3}-631665 x^{2}+420030 x-117575\right) p_{1}^{8}\right) \\
& +\mathcal{O}\left(p_{1}^{10}\right),
\end{aligned}
$$

resulting in

$$
\begin{aligned}
p_{2} & =p_{1}-\frac{1}{9} p_{1}^{3}+\frac{17}{4320} p_{1}^{5}-\frac{1853}{6531840} p_{1}^{7}+\mathcal{O}\left(p_{1}^{9}\right), \\
\lambda & =-1+\frac{1}{24} p_{1}^{2}-\frac{5}{3456} p_{1}^{4}+\frac{43}{414720} p_{1}^{6}-\frac{4703}{501645312} p_{1}^{8}+\mathcal{O}\left(p_{1}^{10}\right) .
\end{aligned}
$$


The choice of $x_{s}$ in (B.7) is motivated so that $\lambda\left(p_{1}=0\right)=-1$. Given (B.15), we can compute perturbatively in $p_{1}$ one-point correlation functions of the stress energy-tensor and $\mathcal{O}_{\phi},(\mathrm{A} .11)-(\mathrm{A} .13)$ :

$$
\begin{aligned}
\frac{1}{c} \frac{\mathcal{E}}{H^{3}} & =\frac{1}{1152} p_{1}^{2}+\frac{5}{82944} p_{1}^{4}-\frac{43}{19906560} p_{1}^{6}+\frac{4703}{30098718720} p_{1}^{8}+\mathcal{O}\left(p_{1}^{10}\right), \\
\frac{1}{c} \frac{P}{H^{3}} & =-\frac{1}{1152} p_{1}^{2}-\frac{5}{82944} p_{1}^{4}+\frac{43}{19906560} p_{1}^{6}-\frac{4703}{30098718720} p_{1}^{8}+\mathcal{O}\left(p_{1}^{10}\right), \\
\frac{1}{c} \frac{\mathcal{O}_{\phi}}{H^{2}} & =\frac{5}{27648} p_{1}^{3}-\frac{43}{6635520} p_{1}^{5}+\frac{4703}{10032906240} p_{1}^{7}+\mathcal{O}\left(p_{1}^{9}\right),
\end{aligned}
$$

where $c$ is the central charge (2.5).

The obtained data sets are used to generate figures 1 and 2 .

\section{B.2 Fluctuations about $\mathbb{Z}_{2}^{\chi}$-symmetric DFP}

The DFP of section B.1 has an unbroken $\mathbb{Z}_{2}^{\chi}$ symmetry. The fluctuations about it decouple into sets, characterized by this symmetry:

- Fluctuations $\left\{\delta \mathcal{E}, \delta P, \delta \mathcal{O}_{\phi}\right\}$ preserve $\mathbb{Z}_{2}^{\chi}$ symmetry, and are stable. They have been extensively studied in [14].

- Fluctuations $\delta \mathcal{O}_{\chi}$ spontaneously break $\mathbb{Z}_{2}^{\chi}$ symmetry. They end up being unstable, provided $\frac{\Lambda}{H}$ is sufficiently large.

The spectrum of $\mathbb{Z}_{2}^{\chi}$ symmetry breaking fluctuations $\delta \mathcal{O}_{\chi}$ is represented by the spectrum of the quasinormal modes of the gravitational scalar $\chi$ in the holographic DFP background (B.2)-(B.5). Introducing

$$
\chi(x, t)=H_{0}(x) e^{-i \hat{\omega} t}, \quad \hat{\omega}=\frac{\omega}{H},
$$

we obtain from the linearization of the third equation in (A.6) about (B.2)-(B.5):

$$
0=H_{0}^{\prime \prime}+\left(\frac{2 \mathcal{F}^{\prime}}{\mathcal{F}}+\frac{\mathcal{G}^{\prime}}{\mathcal{G}}+\frac{2 i \hat{\omega}}{\mathcal{G}}-\frac{2}{x}-\frac{2}{\mathcal{G}}\right) H_{0}^{\prime}+2\left(\frac{i \mathcal{F}^{\prime} \hat{\omega}}{\mathcal{G F}}-\frac{p^{2} g}{\mathcal{G} x^{2}}-\frac{i \hat{\omega}}{\mathcal{G} x}-\frac{2}{\mathcal{G} x^{2}}\right) H_{0}
$$

The spectrum is determined solving for $H_{0}$, subject to the following asymptotics (without the loss of generality we normalized the linearized fluctuations so that $H_{0,4}=1$, see (A.14)):

- in the UV, i.e., as $x \rightarrow 0_{+}$,

$$
H_{0}=x^{4}(\underbrace{H_{0,4}}_{=1}+(4-4 \lambda-i \hat{\omega}) x+\mathcal{O}\left(x^{2}\right)) ;
$$

- in the IR, i.e., as $y \equiv \frac{1}{3}-x \rightarrow 0_{+}$,

$$
H_{0}=\left(\frac{1}{3}-y\right)^{4}\left(H_{0,0}^{s}-\frac{3 H_{0,0}^{s}\left(i \hat{\omega}\left(p_{0}^{s}\right)^{2}+12\left(p_{0}^{s}\right)^{2} g-(10 i) \hat{\omega}+56\right)}{4(i \hat{\omega}-2)} y+\mathcal{O}\left(y^{2}\right)\right) .
$$

Note that there are two parameters characterizing a QNM: $\left\{\hat{\omega}, H_{0,0}^{s}\right\}$ - precisely as needed to solve a single second order ODE (B.18). 
For a finite $p_{1}$, the spectrum is computed numerically, see figure 3 . We can also compute the spectrum perturbatively in $p_{1}$, using (B.12)-(B.14),

$$
\hat{\omega}_{n}=-i n+\mathcal{O}\left(p_{1}^{2}\right), \quad n \in \mathbb{N} \geq 4,
$$

e.g.,

$$
\begin{aligned}
\hat{\omega}_{4}= & -i\left(4+\left(\frac{1}{4} g-\frac{1}{60}\right) p_{1}^{2}+\left(\frac{3}{160} g^{2}-\frac{157}{5400} g+\frac{23}{21600}\right) p_{1}^{4}+\left(-\frac{9073}{108864000}\right.\right. \\
& \left.+\frac{17}{4800} g^{3}+\frac{388879}{108864000} g-\frac{4217}{648000} g^{2}\right) p_{1}^{6}+\left(\frac{7693913}{1097349120000}-\frac{4356421}{2177280000} g^{3}\right. \\
& \left.\left.-\frac{1815343}{4064256000} g+\frac{1621804901}{1097349120000} g^{2}+\frac{2053}{2304000} g^{4}\right) p_{1}^{8}+\mathcal{O}\left(p_{1}^{10}\right)\right),
\end{aligned}
$$

with $n>4$ perturbative spectra readily obtained as well.

\section{DFP with spontaneously broken $\mathbb{Z}_{2}$ symmetry and its fluctuations}

\section{C.1 $\mathbb{Z}_{2}^{\chi}$-broken DFP}

A dynamical fixed point with spontaneously broken $\mathbb{Z}_{2}^{\chi}$ symmetry is the $t \rightarrow \infty$ (late-time) solution of (A.6)-(A.7) with $\chi(t, x) \neq 0$. Introducing

$$
\lim _{t \rightarrow \infty}\{\sigma, a, \phi, \chi\}(t, x)=\left\{\frac{\mathcal{F}(x)}{x}, \frac{\mathcal{G}(x)}{2 x^{2}}, p(x), q(x)\right\},
$$

we find from (A.6)-(A.7)

$$
\begin{aligned}
0= & \mathcal{F}^{\prime \prime}+\frac{1}{4} \mathcal{F}\left(\left(p^{\prime}\right)^{2}+\left(q^{\prime}\right)^{2}\right), \\
0= & p^{\prime \prime}-\frac{2 \mathcal{F} x^{2}}{K}\left(p^{\prime}\right)^{3}-\frac{\mathcal{F} p\left(q^{2} g-1\right)}{K}\left(p^{\prime}\right)^{2}+\frac{1}{K}\left(-2 \mathcal{F} x^{2}\left(q^{\prime}\right)^{2}-\frac{24 x^{2}\left(\mathcal{F}^{\prime}\right)^{2}}{\mathcal{F}}\right. \\
& \left.+\frac{2\left(2 \mathcal{F}^{\prime} x-\mathcal{F}\right)}{x}\left(p^{2} q^{2} g-p^{2}+2 q^{2}\right)+24(x-1) \mathcal{F}^{\prime}+\frac{12 \mathcal{F}}{x}\right) p^{\prime} \\
& +\frac{p\left(-\mathcal{F}^{2}\left(q^{\prime}\right)^{2} x^{2}+12\left(\mathcal{F}^{\prime} x-\mathcal{F}\right)^{2}\right)\left(q^{2} g-1\right)}{\mathcal{F} x^{2} K}, \\
0= & q^{\prime \prime}-\frac{2 \mathcal{F} x^{2}}{K}\left(q^{\prime}\right)^{3}-\frac{\mathcal{F} q\left(p^{2} g+2\right)}{K}\left(q^{\prime}\right)^{2}+\frac{1}{K}\left(-2 \mathcal{F} x^{2}\left(p^{\prime}\right)^{2}-\frac{24 x^{2}\left(\mathcal{F}^{\prime}\right)^{2}}{\mathcal{F}}\right. \\
& \left.+\frac{2\left(2 \mathcal{F}^{\prime} x-\mathcal{F}\right)}{x}\left(p^{2} q^{2} g-p^{2}+2 q^{2}\right)+24(x-1) \mathcal{F}^{\prime}+\frac{12 \mathcal{F}}{x}\right) q^{\prime} \\
& +\frac{q\left(-\mathcal{F}^{2} x^{2}\left(p^{\prime}\right)^{2}+12\left(\mathcal{F}^{\prime} x-\mathcal{F}\right)^{2}\right)\left(p^{2} g+2\right)}{\mathcal{F} x^{2} K},
\end{aligned}
$$

where

$$
K=\mathcal{F}\left(p^{2} q^{2} g-p^{2}+2 q^{2}\right)-12 \mathcal{F}^{\prime} x^{2}+6 \mathcal{F}(2 x-1),
$$

along with the algebraic equation for $\mathcal{G}$ :

$$
\mathcal{G}=\frac{2 \mathcal{F} K}{\mathcal{F}^{2} x^{2}\left(\left(p^{\prime}\right)^{2}+\left(q^{\prime}\right)^{2}\right)-12\left(\mathcal{F}^{\prime} x-\mathcal{F}\right)^{2}} .
$$


The location of the apparent horizon $x_{A H}$ is given by the same expression as (B.6). As for the $\mathbb{Z}_{2}^{\chi}$-symmetric DFP in section $\mathrm{B}$, the vanishing of $K$ (correspondingly $\mathcal{G}$ ) at $x_{s} \in$ $\left(0, x_{A H}\right)$ renderes the equations for the bulk scalars (C.3) and (C.4) singular at $x_{s}$. We use the residual diffeomorphism parameter $\lambda$, see (A.19), to keep the location of $x_{s}$ fixed as in (B.7), as one varies $p_{1}$.

We seek solutions of (C.2)-(C.6) subject to the following boundary conditions:

- in the UV, i.e., as $x \rightarrow 0_{+}$,

$$
\begin{aligned}
\mathcal{F}= & 1+\lambda x-\frac{1}{8} p_{1}^{2} x^{2}+\left(-\frac{1}{24} \lambda p_{1}^{2}-\frac{1}{6} p_{1} p_{2}\right) x^{3}+\mathcal{O}\left(x^{4}\right), \\
p= & p_{1} x+p_{2} x^{2}+\left(\frac{1}{4} p_{1}^{3}-2 \lambda p_{2}+2 p_{2}+2 \lambda p_{1}-\lambda^{2} p_{1}\right) x^{3}+\mathcal{O}\left(x^{4}\right), \\
q= & q_{4} x^{4}+4 q_{4}(1-\lambda) x^{5}+q_{4}\left(\frac{p_{1}^{2} g}{7}+10 \lambda^{2}+\frac{5 p_{1}^{2}}{14}-20 \lambda+\frac{80}{7}\right) x^{6}+\mathcal{O}\left(x^{7}\right), \\
\mathcal{G}= & 1+(-2+2 \lambda) x+\left(-\frac{1}{4} p_{1}^{2}+\lambda^{2}-2 \lambda\right) x^{2}+\left(-\frac{1}{3} \lambda p_{1}^{2}+\frac{1}{3} p_{1}^{2}-\frac{1}{3} p_{1} p_{2}\right) x^{3} \\
& +\left(-\frac{1}{24} p_{1}^{4}-\frac{1}{6} p_{2}^{2}-\frac{1}{3} \lambda p_{1}^{2}+\frac{1}{6} \lambda^{2} p_{1}^{2}\right) x^{4}+\mathcal{O}\left(x^{5}\right)
\end{aligned}
$$

- in the IR, i.e., , as $y \equiv \frac{1}{3}-x \rightarrow 0_{+}$,

$$
\begin{aligned}
\mathcal{F} & =f_{0}^{s}-\frac{3}{4} f_{0}^{s}\left(\left(p_{0}^{s}\right)^{2}\left(q_{0}^{s}\right)^{2} g-\left(p_{0}^{s}\right)^{2}+2\left(q_{0}^{s}\right)^{2}-2\right) y+\mathcal{O}\left(y^{2}\right) \\
p & =p_{0}^{s}+\left(\frac{9}{2} p_{0}^{s}\left(q_{0}^{s}\right)^{2} g-\frac{9}{2} p_{0}^{s}\right) y+\mathcal{O}\left(y^{2}\right) \\
q & =q_{0}^{s}+\left(\frac{9}{2}\left(p_{0}^{s}\right)^{2} q_{0}^{s} g+9 q_{0}^{s}\right) y+\mathcal{O}\left(y^{2}\right) \\
\mathcal{G} & =2 y+\left(\frac{3}{2}\left(p_{0}^{s}\right)^{2}\left(1-\left(q_{0}^{s}\right)^{2} g\right)-3\left(q_{0}^{s}\right)^{2}+3\right) y^{2}+\mathcal{O}\left(y^{3}\right)
\end{aligned}
$$

For a fixed $p_{1}$, alternatively the mass scale $\Lambda$ (A.18), the DFP gravitational dual is characterized by $\left\{p_{2}, q_{4}, \lambda, f_{0}^{s}, p_{0}^{s}, q_{0}^{s}\right\}$ - precisely the number of parameters needed to solve three second order ODEs (C.2)-(C.4). Notice that $\mu$ is not an independent parameter from (A.17) at the DFP $\dot{\mu}=0$ leading to (B.10).

The solutions for $x \in\left[0, \frac{1}{3}\right)$ are then extended for $x \in\left(\frac{1}{3}, x_{A H}\right]$, solving (C.2)-(C.4), with initial conditions (see (C.8))

$$
\begin{aligned}
\mathcal{F} & =f_{0}^{s}-\frac{3}{4} f_{0}^{s}\left(\left(p_{0}^{s}\right)^{2}\left(q_{0}^{s}\right)^{2} g-\left(p_{0}^{s}\right)^{2}+2\left(q_{0}^{s}\right)^{2}-2\right)\left(\frac{1}{3}-x\right)+\mathcal{O}\left(\left(\frac{1}{3}-x\right)^{2}\right) \\
p & =p_{0}^{s}+\left(\frac{9}{2} p_{0}^{s}\left(q_{0}^{s}\right)^{2} g-\frac{9}{2} p_{0}^{s}\right)\left(\frac{1}{3}-x\right)+\mathcal{O}\left(\left(\frac{1}{3}-x\right)^{2}\right) \\
q & =q_{0}^{s}+\left(\frac{9}{2}\left(p_{0}^{s}\right)^{2} q_{0}^{s} g+9 q_{0}^{s}\right)\left(\frac{1}{3}-x\right)+\mathcal{O}\left(\left(\frac{1}{3}-x\right)^{2}\right) \\
\mathcal{G} & =2\left(\frac{1}{3}-x\right)+\left(\frac{3}{2}\left(p_{0}^{s}\right)^{2}\left(1-\left(q_{0}^{s}\right)^{2} g\right)-3\left(q_{0}^{s}\right)^{2}+3\right)\left(\frac{1}{3}-x\right)^{2}+\mathcal{O}\left(\left(\frac{1}{3}-x\right)^{3}\right)
\end{aligned}
$$

while monitoring the AH locator (B.6). 
The data sets $\left\{p_{1}, p_{2}, q_{4}, \lambda, f_{0}^{s}, p_{0}^{s}, q_{0}^{s}\right\}$ are found numerically: $\mathbb{Z}_{2}^{\chi}$-broken DFPs do not extend to $p_{1} \rightarrow 0$, making analytic perturbative analysis impossible. The obtained data sets are used to generate figures 4 and 5 .

\section{C.2 Fluctuations about $\mathbb{Z}_{2}^{\chi}$-broken DFP}

Introducing

$$
\begin{aligned}
\chi & =q(x)+H_{0}(x) e^{-i \hat{\omega} t}, & \phi(t, x) & =p(x)+H_{1}(x) e^{-i \hat{\omega} t}, \\
\sigma(t, x) & =\frac{1}{x}\left(\mathcal{F}(x)+H_{2}(x) e^{-i \hat{\omega} t}\right), & a(t, x) & =\frac{1}{2 x^{2}}\left(\mathcal{G}(x)+H_{3}(x) e^{-i \hat{\omega} t}\right),
\end{aligned}
$$

we obtain from the linearization of (A.6)-(A.7) with respect to $H_{i}$,

$$
\begin{aligned}
0= & H_{0}^{\prime \prime}+\left(\frac{2 i \hat{\omega}}{\mathcal{G}}+\frac{2 \mathcal{F}^{\prime}}{\mathcal{F}}+\frac{\mathcal{G}^{\prime}}{\mathcal{G}}-\frac{2}{x}-\frac{2}{\mathcal{G}}\right) H_{0}^{\prime}+\frac{2 q^{\prime}}{\mathcal{F}} H_{2}^{\prime}+\frac{q^{\prime}}{\mathcal{G}} H_{3}^{\prime}+\left(\left(\frac{2 i \mathcal{F}^{\prime}}{\mathcal{G} \mathcal{F}}-\frac{2 i}{\mathcal{G} x}\right) \hat{\omega}\right. \\
& \left.-\frac{2 p^{2} g}{\mathcal{G} x^{2}}-\frac{4}{\mathcal{G} x^{2}}\right) H_{0}-\frac{4 p q g}{\mathcal{G} x^{2}} H_{1}+\left(\frac{2 i q^{\prime} \hat{\omega}}{\mathcal{G} \mathcal{F}}-\frac{2 q^{\prime} \mathcal{F}}{\mathcal{F}^{2}}\right) H_{2}+\left(\frac{2 p^{2} q g}{\mathcal{G}^{2} x^{2}}-\frac{q^{\prime} \mathcal{G}^{\prime}}{\mathcal{G}^{2}}+\frac{2 q^{\prime}}{\mathcal{G}^{2}}\right. \\
& \left.+\frac{4 q}{\mathcal{G}^{2} x^{2}}\right) H_{3} \\
0 & H_{1}^{\prime \prime}+\left(\frac{2 i \hat{\omega}}{\mathcal{G}}+\frac{2 \mathcal{F}^{\prime}}{\mathcal{F}}+\frac{\mathcal{G}^{\prime}}{\mathcal{G}}-\frac{2}{x}-\frac{2}{\mathcal{G}}\right) H_{1}^{\prime}+\frac{2 p^{\prime}}{\mathcal{F}} H_{2}^{\prime}+\frac{p^{\prime}}{\mathcal{G}} H_{3}^{\prime}-\frac{4 p q g}{\mathcal{G} x^{2}} H_{0} \\
& +\left(\left(\frac{2 i \mathcal{F}^{\prime}}{\mathcal{G F}}-\frac{2 i}{\mathcal{G} x}\right) \hat{\omega}-\frac{2 q^{2} g}{\mathcal{G} x^{2}}+\frac{2}{\mathcal{G} x^{2}}\right) H_{1}+\left(\frac{2 i p^{\prime} \hat{\omega}}{\mathcal{G} \mathcal{F}}-\frac{2 p^{\prime} \mathcal{F}^{\prime}}{\mathcal{F}^{2}}\right) H_{2}+\left(\frac{2 p q^{2} g}{\mathcal{G}^{2} x^{2}}-\frac{p^{\prime} \mathcal{G}^{\prime}}{\mathcal{G}^{2}}\right. \\
& \left.+\frac{2 p^{\prime}}{\mathcal{G}^{2}}-\frac{2 p}{\mathcal{G}^{2} x^{2}}\right) H_{3}, \\
0 & H_{2}^{\prime \prime}+\frac{1}{2} \mathcal{F} q^{\prime} H_{0}^{\prime}+\frac{1}{2} \mathcal{F} p^{\prime} H_{1}^{\prime}+\frac{1}{4}\left(\left(p^{\prime}\right)^{2}+\left(q^{\prime}\right)^{2}\right) H_{2},
\end{aligned}
$$

where we kept $H_{3}$ dependence to avoid cluttering the formulas, although $H_{3}^{\prime}$ and $H_{3}$ can be expressed algebraically through $\left\{H_{0}, H_{1}, H_{2}\right\}$ and their first derivatives:

$$
\begin{aligned}
H_{3}^{\prime}= & \frac{x \mathcal{F} \mathcal{G} q^{\prime}(\mathcal{G}-1)}{2 M} H_{0}^{\prime}+\frac{x \mathcal{F} \mathcal{G} p^{\prime}(\mathcal{G}-1)}{2 M} H_{1}^{\prime}+\frac{1}{M}\left(-\frac{2 \mathcal{G} x(\mathcal{G}-1) \mathcal{F}^{\prime}}{\mathcal{F}}+x(1-\mathcal{G}) \mathcal{G}^{\prime}\right. \\
& \left.+2 i \hat{\omega} x+4 \mathcal{G}^{2}+2 x \mathcal{G}-4 \mathcal{G}-4 x\right) H_{2}^{\prime}+\frac{1}{M}\left(\frac{1}{2} i \mathcal{G} \mathcal{F} \hat{\omega} x q^{\prime}-\frac{q \mathcal{F}\left(p^{2} g+2\right)(\mathcal{G}-1)}{x}\right) H_{0} \\
& +\frac{1}{M}\left(\frac{1}{2} i \mathcal{G} \mathcal{F} \hat{\omega} x p^{\prime}-\frac{p \mathcal{F}\left(q^{2} g-1\right)(\mathcal{G}-1)}{x}\right) H_{1}+\frac{1}{M}\left(\frac{\mathcal{G} x(\mathcal{G}-1)\left(\mathcal{F}^{\prime}\right)^{2}}{\mathcal{F}^{2}}\right. \\
& +\frac{1}{4} \mathcal{G} x(\mathcal{G}-1)\left(\left(p^{\prime}\right)^{2}+\left(q^{\prime}\right)^{2}\right)-\frac{2 i \hat{\omega} x(\mathcal{G}-1) \mathcal{F}^{\prime}}{\mathcal{F}}+(-i \hat{\omega} x+\mathcal{G}+x-1) \mathcal{G}^{\prime}-2 x \hat{\omega}^{2} \\
& \left.+4 i(\mathcal{G}-x-1) \hat{\omega}-\frac{3 \mathcal{G}^{2}}{x}-\frac{\mathcal{G}\left(p^{2}\left(q^{2} g-1\right)+2 q^{2}+8 x-12\right)}{2 x}\right) H_{2}+\frac{1}{M}\left(\frac{1}{4} \mathcal{F} x(2 \mathcal{G}-1)\left(\left(p^{\prime}\right)^{2}+\left(q^{\prime}\right)^{2}\right)\right. \\
& +\frac{\left(p^{2}\left(q^{2} g-1\right)+2 q^{2}+4 x^{2}+8 x-6\right)}{2 x} \\
& -\frac{x(2 \mathcal{G}-1)\left(\mathcal{F}^{\prime}\right)^{2}}{\mathcal{F}}+\left(i \hat{\omega} x-\mathcal{G}^{\prime} x+8 \mathcal{G}+2 x-4\right) \mathcal{F}^{\prime}+\left(\mathcal{F}-x \mathcal{F}^{\prime}\right) \mathcal{G}^{\prime}+x \mathcal{G}^{\prime} \mathcal{F}^{\prime}-i \hat{\omega} \mathcal{F} \\
& \left.-\frac{\mathcal{F}\left(p^{2}\left(q^{2} g-1\right)+2 q^{2}+12 \mathcal{G}+8 x-12\right)}{2 x}\right) H_{3}
\end{aligned}
$$




$$
\begin{aligned}
H_{3}= & \frac{1}{N}\left(-2 \mathcal{G} q^{\prime} \mathcal{F}^{3} x^{3} H_{0}^{\prime}-2 p^{\prime} \mathcal{G} \mathcal{F}^{3} x^{3} H_{1}^{\prime}+\left(8 \mathcal{F} \mathcal{G}(2-i \hat{\omega}) x^{3} \mathcal{F}^{\prime}+4 \mathcal{F}^{2}\left(\mathcal{G}^{\prime} x-6 \mathcal{G}\right.\right.\right. \\
& \left.-4 x+2 i \hat{\omega}(\mathcal{G}+x)) x^{2}\right) H_{2}^{\prime}-2 x \mathcal{F}^{2}\left(i \mathcal{G} q^{\prime}\left(x \mathcal{F}^{\prime}-\mathcal{F}\right) \hat{\omega} x-2 q \mathcal{F}\left(p^{2} g+2\right)\right) H_{0} \\
& -2 x \mathcal{F}^{2}\left(i p^{\prime} \mathcal{G} \hat{\omega} x\left(x \mathcal{F}^{\prime}-\mathcal{F}\right)-2 p\left(q^{2} g-1\right) \mathcal{F}\right) H_{1}+\left(-\mathcal{F}^{2} \mathcal{G} x^{3}\left(\left(p^{\prime}\right)^{2}+\left(q^{\prime}\right)^{2}\right)-4 \mathcal{G} x^{3}\left(\mathcal{F}^{\prime}\right)^{2}\right. \\
& +4 \mathcal{F} x^{3}\left(i \mathcal{G}^{\prime} \hat{\omega}+6 i \hat{\omega}+2 \hat{\omega}^{2}-\mathcal{G}^{\prime}-2\right) \mathcal{F}^{\prime}-4 i \mathcal{F}^{2} x^{2} \hat{\omega} \mathcal{G}^{\prime}-2 x \mathcal{F}^{2}\left(p^{2}\left(1-q^{2} g\right)+16 i \hat{\omega} x\right. \\
& \left.\left.\left.+4 x \hat{\omega}^{2}-2 q^{2}-6 \mathcal{G}-12 x+6\right)\right) H_{2}\right)
\end{aligned}
$$

with

$$
M=x \mathcal{F}^{\prime}(\mathcal{G}-1)-\mathcal{F}(\mathcal{G}+x-1)
$$

and

$$
\begin{aligned}
N= & -4 \mathcal{G} x^{3}\left(\mathcal{F}^{\prime}\right)^{3}+4 \mathcal{F} x^{2}\left(i \hat{\omega} x-\mathcal{G}^{\prime} x+5 \mathcal{G}+x\right)\left(\mathcal{F}^{\prime}\right)^{2}-2 \mathcal{F}^{2} x\left(p^{2} q^{2} g+4 i \hat{\omega} x-p^{2}+2 q^{2}\right. \\
& \left.-4 \mathcal{G}^{\prime} x+14 \mathcal{G}+4 x-6\right) \mathcal{F}^{\prime}+\mathcal{F}^{2} x^{2}\left(x \mathcal{F}^{\prime} \mathcal{G}-\mathcal{F G}+x \mathcal{F}\right)\left(\left(p^{\prime}\right)^{2}+\left(q^{\prime}\right)^{2}\right) \\
& +2 \mathcal{F}^{3}\left(p^{2}\left(q^{2} g-1\right)+2 i \hat{\omega} x+2 q^{2}-2 \mathcal{G}^{\prime} x+6 \mathcal{G}+2 x-6\right)
\end{aligned}
$$

We explicitly verified that (C.14) and (C.15) are consistent, given (C.11)-(C.13) and the background equations (C.2)-(C.6).

The spectrum is determined solving for $H_{i}$, (without the loss of generality we normalized the linearized fluctuations so that $H_{0,4}=1$, see (A.14)):

in the UV, i.e., as $x \rightarrow 0_{+}$,

$$
\begin{aligned}
& H_{0}=x^{4}(\underbrace{H_{0,4}}_{=1}+\left(4-4 H_{2,1} q_{4}-i \hat{\omega}-4 \lambda\right) x+\mathcal{O}\left(x^{2}\right)) \\
& H_{1}=H_{1,2} x^{2}+\left(\left(2 p_{1}-i \hat{\omega} p_{1}-2 \lambda p_{1}-2 p_{2}\right) H_{2,1}+(2-i \hat{\omega}-2 \lambda) H_{1,2}\right) x^{3}+\mathcal{O}\left(x^{4}\right) \\
& H_{2}=H_{2,1} x+\left(-\frac{p_{1}}{6} H_{1,2}-\frac{p_{1}^{2}}{24} H_{2,1}\right) x^{3}+\mathcal{O}\left(x^{4}\right)
\end{aligned}
$$

- in the IR, i.e., as $y \equiv \frac{1}{3}-x \rightarrow 0_{+}$,

$$
\begin{aligned}
H_{0}= & H_{0,0}^{s}+\frac{3}{4 f_{0}^{s}(i \hat{\omega}-2)}\left(\left(i f_{0}^{s}\left(\left(p_{0}^{s}\right)^{2}\left(\left(q_{0}^{s}\right)^{2} g-1\right)+2\left(q_{0}^{s}\right)^{2}-6\right) \hat{\omega}-12 f_{0}^{s}\left(\left(p_{0}^{s}\right)^{2} g\right.\right.\right. \\
& \left.+2)) H_{0,0}^{s}-24 f_{0}^{s} p_{0}^{s} q_{0}^{s} g H_{1,0}^{s}-\left(6 q_{0}^{s}\left(\left(p_{0}^{s}\right)^{2} g+2\right) \hat{\omega}^{2}+12 i q_{0}^{s}\left(\left(p_{0}^{s}\right)^{2} g+2\right) \hat{\omega}\right) H_{2,0}^{s}\right) y \\
& +\mathcal{O}\left(y^{2}\right), \\
H_{1}= & H_{1,0}^{s}+\frac{3}{4 f_{0}^{s}(i \hat{\omega}-2)}\left(-24 f_{0}^{s} p_{0}^{s} q_{0}^{s} g H_{0,0}^{s}+\left(i f_{0}^{s}\left(\left(p_{0}^{s}\right)^{2}\left(q_{0}^{s}\right)^{2} g-\left(p_{0}^{s}\right)^{2}+2\left(q_{0}^{s}\right)^{2}-6\right) \hat{\omega}\right.\right. \\
& \left.\left.-12 f_{0}^{s}\left(\left(q_{0}^{s}\right)^{2} g-1\right)\right) H_{1,0}^{s}-\left(6 p_{0}^{s}\left(\left(q_{0}^{s}\right)^{2} g-1\right) \hat{\omega}^{2}+12 i p_{0}^{s}\left(\left(q_{0}^{s}\right)^{2} g-1\right) \hat{\omega}\right) H_{2,0}^{s}\right) y \\
& +\mathcal{O}\left(y^{2}\right),
\end{aligned}
$$




$$
\begin{aligned}
H_{2}= & H_{2,0}^{s}+\frac{3}{4 f_{0}^{s}(i \hat{\omega}-3)}\left(6\left(f_{0}^{s}\right)^{2} q_{0}^{s}\left(\left(p_{0}^{s}\right)^{2} g+2\right) H_{0,0}^{s}+\left(6 p_{0}^{s}\left(\left(q_{0}^{s}\right)^{2} g-1\right)\left(f_{0}^{s}\right)^{2}\right) H_{1,0}^{s}\right. \\
& +\left(f_{0}^{s} \hat{\omega}^{2}\left(\left(\left(q_{0}^{s}\right)^{2} g-1\right)\left(p_{0}^{s}\right)^{2}+2\left(q_{0}^{s}\right)^{2}-6\right)+(2 i) f_{0}^{s}\left(\left(\left(q_{0}^{s}\right)^{2} g-1\right)\left(p_{0}^{s}\right)^{2}+2\left(q_{0}^{s}\right)^{2}-8\right) \hat{\omega}\right. \\
& \left.\left.+3 f_{0}^{s}\left(\left(\left(q_{0}^{s}\right)^{2} g-1\right)\left(p_{0}^{s}\right)^{2}+2\left(q_{0}^{s}\right)^{2}-2\right)\right) H_{2,0}^{s}\right) y+\mathcal{O}\left(y^{2}\right) .
\end{aligned}
$$

Note that there are 6 parameters characterizing a QNM:

$$
\left\{\hat{\omega}, H_{1,2}, H_{2,1}, H_{0,0}^{s}, H_{1,0}^{s}, H_{2,0}^{s}\right\}
$$

precisely as needed to solve three second order ODE (C.11)-(C.13).

The unstable QNM is computed numerically, and is presented in figure 6.

\section{Dynamical entropy and the entanglement entropy of a DFP}

\section{D.1 Dynamical entropy from the holographic dual}

One of the advantages of the holographic formulation of a QFT dynamics is the natural definition of its far-from-equilibrium entropy density. A gravitational geometry (2.8) has an apparent horizon located at $r=r_{A H}$, where [19]

$$
\left.d_{+} \Sigma\right|_{r=r_{A H}}=0
$$

Following $[25,26]$ we associate the non-equilibrium entropy density $s$ of the boundary QFT with the Bekenstein-Hawking entropy density of the apparent horizon

$$
e^{2 H \tau} s=\left.\frac{2 \pi}{\kappa^{2}} \Sigma^{2}\right|_{r=r_{A H}}
$$

Using the holographic background equations of motion (A.1)-(A.3) we find

$$
\frac{d\left(e^{2 H \tau} s\right)}{d \tau}=\left.\frac{2 \pi}{\kappa^{2}}\left(\Sigma^{2}\right)^{\prime} \frac{\left(d_{+} \phi\right)^{2}+\left(d_{+} \chi\right)^{2}}{\phi^{2}+6-2 \chi^{2}-g \phi^{2} \chi^{2}}\right|_{r=r_{A H}}
$$

Following [11] it is easy to prove that the comoving entropy production rate as defined by (D.3) is non-negative, i.e.,

$$
\frac{d\left(e^{2 H \tau} s\right)}{d \tau} \geq 0
$$

in holographic dynamics governed by (A.1)-(A.3):

- In (A.10) we showed that $\partial_{x} \sigma<0$, thus

$$
\partial_{r} \Sigma>0
$$

for all times. 

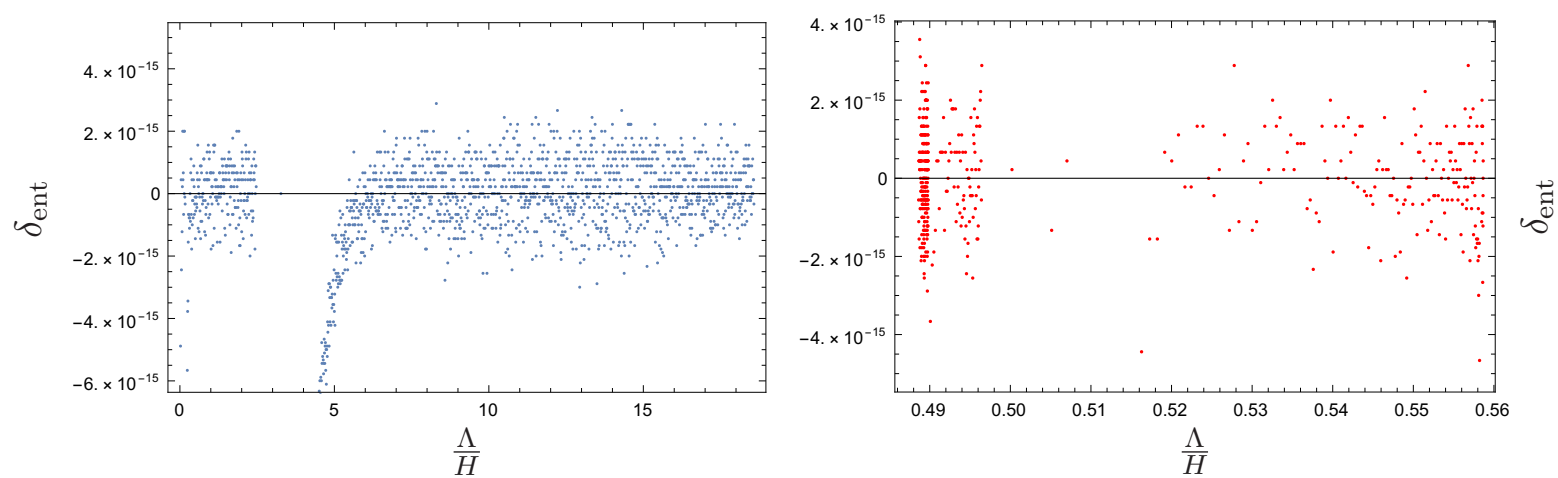

Figure 17. Comparison of the vacuum entanglement entropy density of the $\mathrm{DFP}_{s}$ (the left panel) and the $\mathrm{DFP}_{b}$ (the right panel) computed from (D.9) and (D.10). See (D.11) for the definition of $\delta_{\text {ent }}$.

- Apparent horizon is defined as the innermost (with respect to the boundary) coordinate location $r=r_{A H}$, where $d_{+} \Sigma(\tau, r)$ vanishes. Notice from (A.8),

$$
d_{+} \Sigma(\tau, r)=\frac{e^{H \tau} r^{2}}{2}+\mathcal{O}(r)>0, \quad r \rightarrow \infty .
$$

Thus, assuming the analyticity of the background geometry,

$$
d_{+} \Sigma(\tau, r)>0, \quad r>\left.r_{A H} \quad \Longrightarrow \quad\left(d_{+} \Sigma\right)^{\prime}(\tau, r)\right|_{r=r_{A H}} \geq 0
$$

The first evolution equation in (A.1), evaluated at the apparent horizon, i.e., when $d_{+} \Sigma=0$, reads

given (D.7).

$$
\begin{gathered}
0=\left(d_{+} \Sigma\right)^{\prime}-\left.\frac{\Sigma}{4}\left(\phi^{2}+6-2 \chi^{2}-g \phi^{2} \chi^{2}\right)\right|_{r=r_{A H}+0} \\
\left.\Longrightarrow \quad\left(\phi^{2}+6-2 \chi^{2}-g \phi^{2} \chi^{2}\right)\right|_{r=r_{A H}+0} \geq 0,
\end{gathered}
$$

- Combining (D.5) and (D.8), we arrive from (D.3) to (D.4).

\section{D.2 Entanglement entropy density of a DFP}

Following [13], the entanglement entropy density of a dynamical fixed point $s_{\text {ent }}$ is simply the late-time limit of the non-equilibrium entropy density (D.2): ${ }^{11}$

$$
s_{\text {ent }} \equiv \lim _{\tau \rightarrow \infty} s=\left.\frac{2 \pi H^{2}}{\kappa^{2}}\left(\frac{\mathcal{F}(x)}{x}\right)^{2}\right|_{x=x_{A H}},
$$

where we used (C.1) for the late-time limit of $\sigma(t, x)$.

Alternatively, the DFP entanglement entropy density can be compute from (D.3)

$$
\begin{aligned}
s_{\mathrm{ent}} & =\left.\lim _{\tau \rightarrow \infty} \frac{2 \pi}{\kappa^{2}} \frac{\left(\Sigma^{2}\right)^{\prime}}{2 H e^{2 H \tau}} \frac{\left(d_{+} \phi\right)^{2}+\left(d_{+} \chi\right)^{2}}{\phi^{2}+6-2 \chi^{2}-g \phi^{2} \chi^{2}}\right|_{r=r_{A H}} \\
& =\frac{2 \pi H^{2}}{\kappa^{2}}\left[\left(-x^{2} \partial_{x}\right)\left(\frac{\mathcal{F}(x)}{x}\right)^{2}\right] \times\left.\frac{\left(-\frac{1}{2} \mathcal{G} p^{\prime}\right)^{2}+\left(-\frac{1}{2} \mathcal{G} q^{\prime}\right)^{2}}{p^{2}+6-2 q^{2}-g p^{2} q^{2}}\right|_{x=x_{A H}} .
\end{aligned}
$$

\footnotetext{
${ }^{11}$ See also $[12,22]$.
} 
An agreement of $s_{\mathrm{ent}}^{(\mathrm{D} .9)}$ and $s_{\mathrm{ent}}^{(\mathrm{D} .10)}$ is a highly nontrivial check on our numerics. In figure 17 we plot

$$
\delta_{\mathrm{ent}} \equiv \frac{s_{\mathrm{ent}}^{(\mathrm{D} .9)}}{s_{\mathrm{ent}}^{(\mathrm{D} .10)}}-1,
$$

as a function of $p_{1}$ for the $\mathbb{Z}_{2}^{\chi}$-symmetric DFP (the left panel) and for the symmetry-broken DFP (the right panel).

Given an analytic solution of the gravitational dual to the $\mathbb{Z}_{2}^{\chi}$-symmetric DFP in the limit $p_{1} \rightarrow 0$, see (B.12)-(B.14), we find from (B.6) the location of the apparent horizon as

$$
x_{A H}=1-\frac{1}{6} 6^{2 / 3} p_{1}^{2 / 3}+\frac{1}{12} 6^{1 / 3} p_{1}^{4 / 3}+\frac{1}{9} p_{1}^{2}-\frac{1801}{38880} 6^{2 / 3} p_{1}^{8 / 3}+\mathcal{O}\left(p_{1}^{10 / 3}\right) .
$$

Either from (D.9) or (D.10) we compute

$$
\frac{s_{\text {ent }}}{4 \pi c H^{2}}=\frac{1}{2304} 6^{1 / 3} p_{1}^{4 / 3}-\frac{1}{4608} p_{1}^{2}-\frac{5}{82944} 6^{2 / 3} p_{1}^{8 / 3}+\frac{79}{1866240} 6^{1 / 3} p_{1}^{10 / 3}+\mathcal{O}\left(p_{1}^{4}\right) .
$$

\section{E Numerical setup}

We adapt the characteristic formulation of [19] for the numerical solution of (A.1)-(A.3).

\section{E.1 Field redefinitions and the code equations}

We redefine the fields

$$
\left\{\phi, \chi, \sigma, a, d_{+} \phi, d_{+} \chi, d_{+} \sigma\right\} \rightarrow\{p, q, s, \mathcal{A}, d p, d q, d s\}
$$

as follows

$$
\begin{aligned}
\phi(t, x) & =x p_{1}+x p(t, x), \\
\chi(t, x) & =x^{3} q(t, x), \\
\sigma(t, x) & =\frac{1}{x}+s(t, x), \\
a & =\frac{1}{2} \sigma(t, x)^{2}-\sigma(t, x)+\mathcal{A}(t, x), \\
d_{+} \phi(t, x) & =-\frac{p_{1}}{2}+x d p(t, x), \\
d_{+} \chi(t, x) & =x^{3} d q(t, x), \\
d_{+} \sigma(t, x) & =x d s(t, x)+\frac{1}{2} \sigma(t, x)^{2}-\sigma(t, x)+\frac{p_{1}^{2}}{16} .
\end{aligned}
$$

Using (A.8), we find the asymptotic boundary expansion $x \rightarrow 0_{+}$for the new fields:

$$
\begin{array}{rlrl}
p & =p_{2}(t) x+\mathcal{O}\left(x^{2}\right), & q & =q_{4}(t) x+\mathcal{O}\left(x^{2}\right), \\
d p & =-p_{2}(t)-p_{1} \lambda(t)+p_{1}+\mathcal{O}(x), & d q & =-2 q_{4}(t)+\mathcal{O}(x), \\
s & =\lambda(t)-\frac{p_{1}^{2}}{8} x+\mathcal{O}\left(x^{2}\right), & d s=\mu(t)-\frac{p_{1}^{2}}{12}+\frac{p_{1}}{12} p_{2}(t)+\frac{p_{1}^{2}}{12} \lambda(t)+\mathcal{O}(x), \\
\mathcal{A} & =-\dot{\lambda}(t)+\left(\mu(t)+\frac{p_{1}^{2}}{12}-\frac{p_{1}}{12} p_{2}(t)-\frac{p_{1}^{2}}{12} \lambda(t)\right) x+\mathcal{O}\left(x^{2}\right) .
\end{array}
$$


In new variables (E.2), the equations of motion used to evolve the system take form:

$$
\begin{aligned}
& {\left[\partial_{x x}^{2}+\frac{2}{x} \partial_{x}+\frac{x^{4}}{4}\left(3 q+x q^{\prime}\right)^{2}+\frac{1}{4}\left(p_{1}+p+x p^{\prime}\right)^{2}\right] s=J_{s},} \\
& J_{s}\left\{p, p^{\prime}, q, q^{\prime}\right\}=-\frac{x^{3}}{4}\left(3 q+x q^{\prime}\right)^{2}-\frac{1}{4 x}\left(x p^{\prime}+p+p_{1}\right)^{2}, \\
& {\left[\partial_{x}+\frac{x s^{\prime}+s}{1+x s}\right] d s=J_{d s}} \\
& J_{d s}\left\{p, q, s, s^{\prime}\right\}=-\frac{s^{\prime}}{s x+1}\left(\frac{3}{2} s^{2}+\frac{1}{16} p_{1}^{2}+\frac{3 s}{x}+\frac{3}{2 x^{2}}\right)-\frac{(s x+1)\left(p_{1}+p\right)^{2}}{4 x^{2}} \\
& +\frac{p_{1}^{2}}{16 x^{2}(s x+1)}+\frac{1}{4}\left(g x^{2}\left(p_{1}+p\right)^{2}+2\right)(1+s x) q^{2} x^{2}, \\
& {\left[\partial_{x}+\frac{x s^{\prime}+s}{1+x s}\right] d p+\left[\frac{x\left(p_{1}+p+x p^{\prime}\right)}{1+x s}\right] d s=J_{d p},} \\
& J_{d p}\left\{p, p^{\prime}, q, s, s^{\prime}\right\}=-\frac{p^{\prime}}{16(s x+1)}\left(8 s^{2} x+p_{1}^{2} x+16 s+\frac{8}{x}\right)+\frac{p_{1} s^{\prime}}{2(s x+1)} \\
& -\frac{1}{16(s x+1)}\left(8 s^{2} p+8 s^{2} p_{1}+p p_{1}^{2}+p_{1}^{3}-\frac{8 p}{x^{2}}\right)-\left(p_{1}+p\right) q^{2} x^{4} g, \\
& {\left[\partial_{x}+\frac{2+3 x s+x^{2} s^{\prime}}{x(1+x s)}\right] d q+\left[\frac{x\left(q^{\prime} x+3 q\right.}{1+x s}\right] d s=J_{d q},} \\
& J_{d q}\left\{p, q, q^{\prime}, s\right\}=-\left(p_{1}+p\right)^{2} q g-\frac{q^{\prime}}{16 x(1+s x)}\left(8 s^{2} x^{2}+p_{1}^{2} x^{2}+16 s x+8\right) \\
& -\frac{q}{16(1+s x) x^{2}}\left(24 s^{2} x^{2}+3 p_{1}^{2} x^{2}+80 s x+56\right), \\
& {\left[\partial_{x x}^{2}+\frac{2}{x} \partial_{x}\right] \mathcal{A}+\left[\frac{2\left(x^{2} s^{\prime}-1\right)}{x(1+x s)^{2}}\right] d s-\left[\frac{p_{1}+p+x p^{\prime}}{2 x}\right] d p-\left[\frac{x^{3}\left(x q^{\prime}+3 q\right)}{2}\right] d q=J_{\mathcal{A}},} \\
& J_{\mathcal{A}}\left\{p, p^{\prime}, q, q^{\prime}, s, s^{\prime}\right\}=\frac{1}{4}(s x+1)(s x-x+1)\left(p^{\prime}\right)^{2}-\left(\frac{x s(1-s)\left(p+p_{1}\right)}{2}\right. \\
& \left.-\frac{\left(p_{1}+p\right)(2 s-1)}{2}-\frac{2 p+p_{1}}{4 x}\right) p^{\prime}-\left(s^{\prime}\right)^{2}+\frac{s^{\prime}}{8(s x+1)^{2}}\left(8 s^{2}+\frac{8}{x^{2}}-p_{1}^{2}+\frac{16 s}{x}\right) \\
& +\frac{s\left(p_{1}+p\right)^{2}}{4}\left(s-1+\frac{2}{x}\right)-\frac{\left(p_{1}+p\right)\left(x p+p_{1} x-p\right)}{4 x^{2}}+\frac{p_{1}^{2}}{8 x^{2}(s x+1)^{2}} \\
& +\frac{1}{4} x^{2}\left(q^{\prime} x+3 q\right)^{2}(1+s x)(s x-x+1), \\
& \dot{p}=d p-\frac{p_{1}}{2 x}+\frac{p_{1}+p+x p^{\prime}}{2}\left(x\left(s^{2}-2 s+2 \mathcal{A}\right)+2 s-2+\frac{1}{x}\right), \\
& \dot{q}=d q+\left(x\left(\frac{1}{2} s^{2}-s+\mathcal{A}\right)+s-1+\frac{1}{2 x}\right)\left(q^{\prime} x+3 q\right), \\
& \dot{\mu}=\frac{1}{4} \lambda p_{1}^{2}+\frac{1}{4} p_{1} p_{2}-3 \mu \text {. }
\end{aligned}
$$

Numerical code is organized as follows. 
- [Step 1]: assume that at a time step $t$ we have profiles

$$
\begin{aligned}
& \left\{p(t, x), q(t, x), p^{\prime}(t, x), q^{\prime}(t, x)\right\} \quad \text { and } \\
& \left\{\lambda(t), \mu(t), p_{2}(t) \equiv p^{\prime}(t, x=0), q_{4}(t) \equiv q^{\prime}(t, x=0)\right\} .
\end{aligned}
$$

- [Step 2]: we solve linear in $s$ equation (E.4), subject to boundary conditions

$$
s(t, x=0)=\lambda(t), \quad s^{\prime}(t, x=0)=-\frac{p_{1}^{2}}{8} .
$$

- [Step 3]: we solve linear in $d s$ equation (E.5), subject to the boundary conditions

$$
d s(t, x=0)=\mu(t)-\frac{p_{1}^{2}}{12}+\frac{p_{1}}{12} p_{2}(t)+\frac{p_{1}^{2}}{12} \lambda(t) .
$$

- [Step 4]: we solve linear in $d p$ equation (E.6), subject to the boundary conditions

$$
d p(t, x=0)=-p_{2}(t)-\lambda(t) p_{1}+p_{1} .
$$

- [Step 5]: we solve linear in $d q$ equation (E.7), subject to the boundary conditions

$$
d q(t, x=0)=-2 q_{4}(t) .
$$

- [Step 6]: we solve linear in $a$ equation (E.8), subject to the boundary conditions

$$
\mathcal{A}^{\prime}(t, x=0)=\mu(t)+\frac{p_{1}^{2}(1-\lambda(t))}{12}-\frac{p_{1}}{12} p_{2}(t), \quad \mathcal{A}(t, x=1)=a^{h} .
$$

The value $a^{h}$ is determined from the stationarity of the apparent horizon at $x=1$ as explained in the following subsection.

- [Step7]: we use evolution equations (E.9), along with (see (E.3))

$$
\dot{\lambda}(t)=-\mathcal{A}(t, x=0),
$$

to compute

$$
\{p(t+d t, x), q(t+d t, x), \lambda(t+d t), \mu(t+d t)\} .
$$

After computing the radial coordinate derivatives $\left\{p^{\prime}(t+d t, x), q^{\prime}(t+d t, x)\right\}$, we repeat [Step 1].

Notice that the first equation in (A.6) is redundant in our numerical procedure: rather than propagating in time $\sigma$, we compute it from the constraint (A.7) at each time step; nonetheless, we monitor the consistency of that equation during the evolution.

Implementing the code, ${ }^{12}$ we use spectral methods for the radial coordinate integration, [Step 2]-[Step 6]. Singularities of the equations at the boundary collocation point $x=0$ are resolved using the corresponding boundary conditions instead. We use fourth-order Runge-Kutta method for the time evolution, [Step 7].

\footnotetext{
${ }^{12}$ Code implementation is similar to the one used in [10].
} 


\section{E.2 Apparent horizon and the boundary condition for $a$}

Our numerical implementation requires an independent computation of $a^{h} \equiv \mathcal{A}(t, x=$ 1) (see (E.15)), given radial profiles $\left\{p, p^{\prime}, q, q^{\prime}, s, s^{\prime}, d p, d s, d q\right\}$ and the diffeomorphism parameter $\lambda$ at time $t$. Following [19], this is done by enforcing the time-independent location of the horizon. Apparent horizon is located as $x=x_{A H}$ such that, see (A.20),

$$
\left.\left(d_{+} \sigma(t, x)+\sigma(t, x)\right)\right|_{x=x_{A H}}=0
$$

Assuming $x_{A H}=1, \frac{d x_{A H}}{d t}=0$, and using equations of motion (E.4)-(E.9) we compute $a^{h}$ from

$$
\left.\partial_{t}\left(d_{+} \sigma\left(t, x_{A H}\right)+\sigma\left(t, x_{A H}\right)\right)\right|_{x_{A H}=1}=0 .
$$

Denoting

$$
\left.\left\{p^{h}, d p^{h}, q^{h}, d q^{h}, s^{h}\right\} \equiv\{p, d p, q, d q, s\}\right|_{(t, x=1)},
$$

we find

$$
a^{h}=-\frac{1}{2}\left(s^{h}\right)^{2}+\frac{1}{2}+\frac{\left(d q^{h}\right)^{2}+\left(d p^{h}-\frac{1}{2} p_{1}\right)^{2}}{\left(\left(q^{h}\right)^{2} g-1\right)\left(p^{h}+p_{1}\right)^{2}+2\left(q^{h}\right)^{2}-6} .
$$

\section{E.3 Initial conditions}

To evolve (E.4)-(E.9) one has to provide data, at $t=0$ as required by [Step 1], see (E.10). In particular, we need to specify $\lambda_{0} \equiv \lambda(t=0)$. Once again, we follow [19].

Recall that both $\phi$ and $\chi$ are left invariant under the reparametrization transformations:

$$
\frac{1}{x} \rightarrow \frac{1}{x}+\lambda_{0}
$$

To maintain this invariance, we specify initial conditions for $\{p, q\}$ (in $\lambda_{0}$-invariant way) in terms of two amplitudes $\left\{\mathcal{A}_{p}, \mathcal{A}_{q}\right\}$ :

$$
\begin{aligned}
& \left.p\right|_{t=0}=\mathcal{A}_{p} \frac{x}{\left(1+x \lambda_{0}\right)^{2}} \exp \left[-\frac{x}{1+x \lambda_{0}}\right]-\frac{p_{1} \lambda_{0} x}{1+x \lambda_{0}}, \\
& \left.q\right|_{t=0}=\mathcal{A}_{q} \frac{x}{\left(1+x \lambda_{0}\right)^{4}} \exp \left[-\frac{x}{1+x \lambda_{0}}\right]
\end{aligned}
$$

We then proceed as follows: ${ }^{13}$

- given $\left\{\mathcal{A}_{p}, \mathcal{A}_{q}\right\}$ we set $\lambda_{0}=0$ and perform [Step 2] (E.11) and [Step 3] (E.12);

- having enough data, we follow (E.2) to compute the profile $d_{+} \sigma(t=0, x)$;

- we find numerically the root $x=x_{0}$ of the equation

$$
\left.\left(d_{+} \sigma(t=0, x)+\sigma(t=0, x)\right)\right|_{x=x_{0}}=0
$$

\footnotetext{
${ }^{13}$ For this procedure the integration range over the radial coordinate $x$ might exceed unity.
} 
- we set the trial value of $\lambda_{0}$ as

$$
\lambda_{0}=\frac{1}{x_{0}}-1
$$

which (apart from the numerical errors) would guarantee that the corresponding location of the apparent horizon is now at $x=1$;

- the trial value (E.25) is further adjusted repeatedly performing [Step 2] and [Step 3] to achieve (E.24) at a high accuracy.

\section{E.4 DFP with spontaneously broken $\mathbb{Z}_{2}$ symmetry as an initial condition}

Gravitational dual to a DFP with spontaneously broken $\mathbb{Z}_{2}$ symmetry, see section C, can be introduced directly into a numerical code of section $\mathrm{E}$ as follows:

- We need to set up [Step 1] by providing the profiles for $p_{c}(t=0, x), q_{c}(t=0, x)$. We used the subscript $c$ to differentiate these fields from the related profiles of a DFP, see (C.1), $p(z)$ and $q(z)$. Note that we relabeled the radial coordinate used in section $\mathrm{C}$ as $x \rightarrow z$. The reason for this is that in the evolution code the radial coordinate varies $x \in[0,1]$ (with the fixed location $x=1$ for the apparent horizon), while in computing the DFP, the radial coordinate varies as $z \in\left[0, \frac{1}{3}\right) \cup\left(\frac{1}{3}, x_{A H}\right]$. We remind the reader that the reason we are forced to split the integration range in determining the DFP profiles in section $\mathrm{C}$ is due to the fact that the corresponding equations (C.2)-(C.4) have a coordinate (not physical) singularity whenever $a(t, z)$ vanishes: $a\left(t, z=x_{s}\right)=0, x_{s} \in\left(0, x_{A H}\right)$. As done in section $\mathrm{C}$, it is convenient to keep $x_{s}$ fixed (our choice is $x_{s}=\frac{1}{3}$ ) while to allow for a variation of $x_{A H}$ as one changes $p_{1}$, correspondingly $\Lambda$ in (2.6).

- Radial coordinates used in sections $\mathrm{C}$ and $\mathrm{E}$ are related by a simple coordinate diffeomorphism (A.19):

$$
x=\frac{z}{1+\delta z}, \quad \delta=1-\frac{1}{x_{A H}} .
$$

- The gravitational scalars $\phi$ and $\chi$ are invariant under the coordinate diffeomorphism (A.19); thus, recall (E.2),

$$
\begin{array}{ll}
\left.\phi\right|_{t=0}=p_{1} x+x p_{c}(0, x)=p\left(\frac{x}{1-\delta x}\right), & x \in[0,1], \\
\left.\chi\right|_{t=0}=x^{3} q_{c}(0, x)=q\left(\frac{x}{1-\delta x}\right), & x \in[0,1] .
\end{array}
$$

Given Wolfram Mathematica computed profiles $p(z)$ and $q(z)$, it is straightforward to use (E.26) and (E.27) to output the profiles $p_{c}(0, x)$ and $q_{c}(0, x)$ at collocation points, suitable for FORTRAN code used in section E.

- The diffeomorphism parameters $\lambda_{c}(t=0)$ (used in section E) and the corresponding parameter $\lambda$ (used in section $\mathrm{C}$ ) are related as

$$
\lambda_{c}(t=0)=\lambda-\delta .
$$



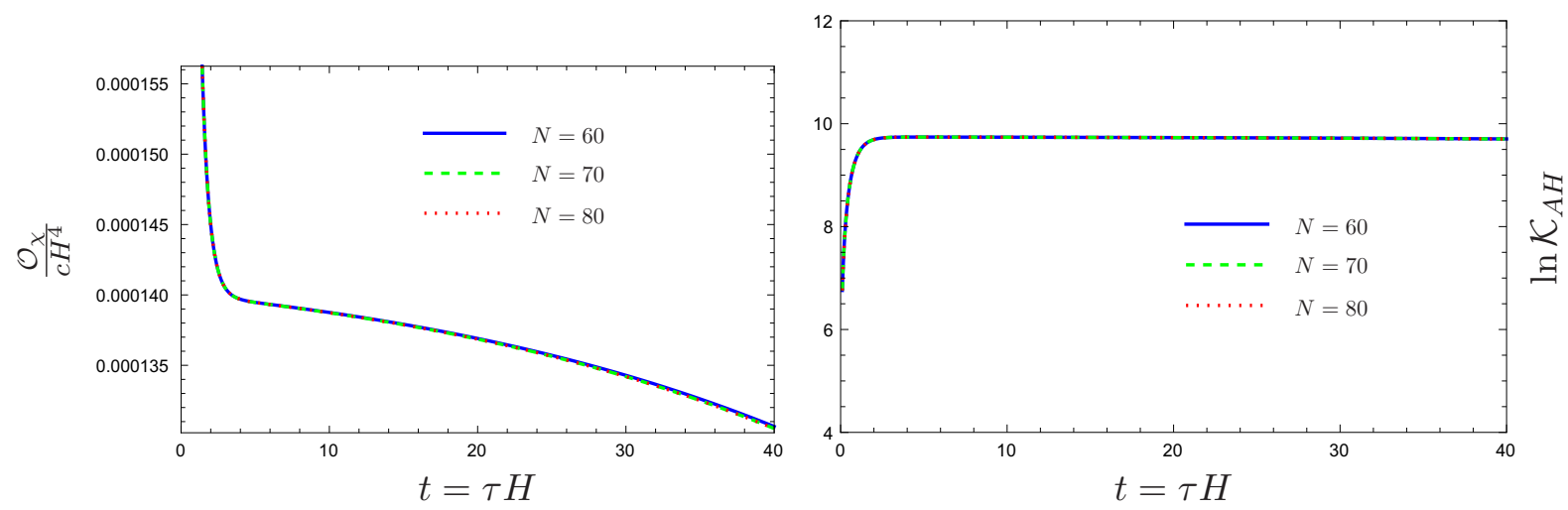

Figure 18. Convergence of simulations performed at different collocation points $N$ with a DFP attractor at late times. Here, $\frac{\Lambda}{H}=p_{1}^{*}$, see (3.13).

- From (E.27),

$$
p_{2, c}(t=0)=p_{2}+p_{1} \delta .
$$

where again we used the subscript $c$ to differentiate the corresponding parameters used in sections $\mathrm{E}$ and $\mathrm{C}$.

- To complete set up of [Step 1] we need $\mu_{c}(t=0)$. Since the input is a DFP,

$$
\dot{\mu}_{c}(t=0)=0
$$

thus, from (E.9)

$$
\mu_{c}(t=0)=\frac{1}{12} \lambda_{c} p_{1}^{2}+\frac{1}{12} p_{1} p_{2, c}=\frac{1}{12} p_{1}\left(p_{2, c}+\lambda_{c} p_{1}\right)=\frac{1}{12} p_{1}\left(p_{2}+\lambda p_{1}\right) ，
$$

where to arrive at the last equality we used (E.29).

The manipulations described above necessarily introduce numerical noise - which is important given that $\mathbb{Z}_{2}$ spontaneously broken DFPs are perturbatively unstable.

\section{E.5 Convergence tests}

We performed self-convergence tests to verify the validity of the obtained numerical solutions. In particular, we study each configuration numerically under different number of collocation points $N=60,70,80$. We monitored the convergence of the residuals of the constraint equations to zero as well as each evolved field (and computing self-convergence test by a suitable interpolation onto a finite difference grid). Additionally, we confirmed convergence of the Kretschmann scalar $\mathcal{K}$ at the apparent horizons, $\mathcal{K}_{A H}=\mathcal{K}\left(t, x_{A H}\right)$ :

$$
\begin{aligned}
\mathcal{K}(t, x) \equiv R_{\mu \nu \rho \lambda} R^{\mu \nu \rho \lambda}= & x^{4}\left(\chi^{\prime} d_{+} \phi+\phi^{\prime} d_{+} \chi\right)^{2}+2 x^{4}\left(\left(\chi^{\prime} d_{+} \chi\right)^{2}+\left(\phi^{\prime} d_{+} \phi\right)^{2}\right) \\
& +\left(\phi^{2} \chi^{2} g-\phi^{2}+2 \chi^{2}-6\right)^{2},
\end{aligned}
$$

where we used the equations of motion (A.6) and (A.7). As an illustration, figures 18 and 19 display the expectation values $\mathcal{O}_{\chi}(t)$ and $\mathcal{K}_{\mathrm{AH}}(t)$ for both the stable and unstable 

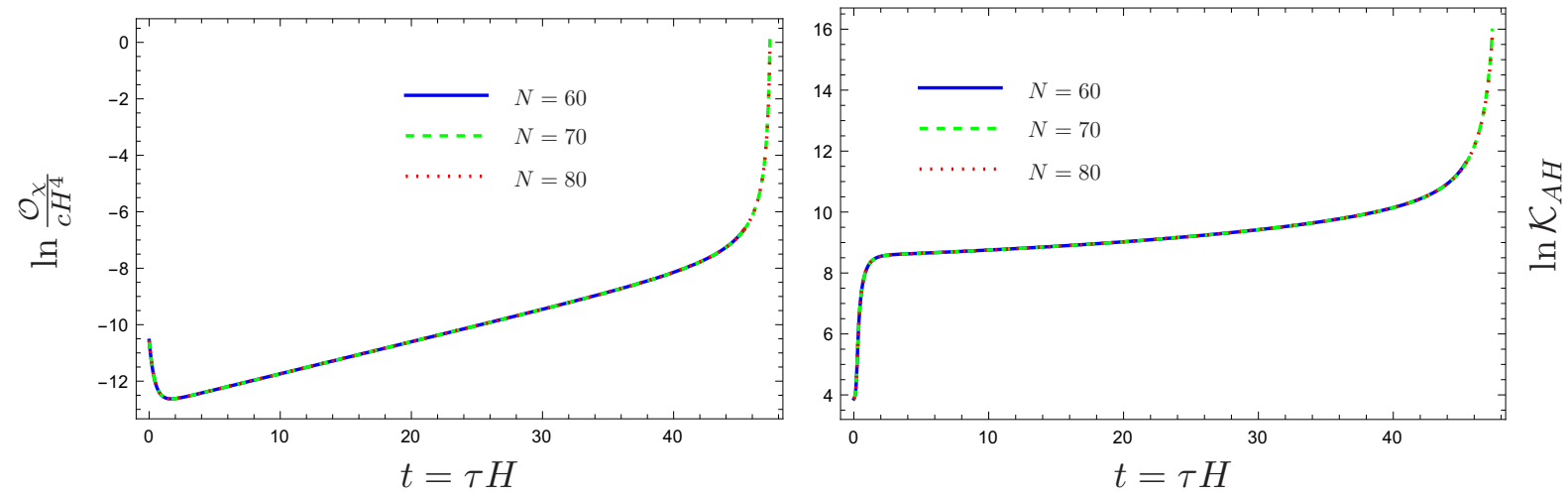

Figure 19. Convergence of simulations performed at different collocation points $N$ without an attractor at late times. Here, $\frac{\Lambda}{H}=\tilde{p}_{1}$, see (3.17).

configurations correspondingly. The fractional difference between the time series of the observables (A.11)-(A.14), e.g.,

$$
\max _{t}\left|\frac{\mathcal{O}^{N_{2}}(t)}{\mathcal{O}^{N_{1}}(t)}-1\right|
$$

for the runs with $N_{1}=70$ and $N_{2}=80$ collocation points is $\sim 10^{-6}-10^{-5}$, both for stable and unstable configurations. All the numerical runs reported in the body of the paper are performed with $N=80$ collocation points.

Open Access. This article is distributed under the terms of the Creative Commons Attribution License (CC-BY 4.0), which permits any use, distribution and reproduction in any medium, provided the original author(s) and source are credited.

\section{References}

[1] T. Dauxois, Fermi, Pasta, Ulam, and a mysterious lady, Phys. Today 61 (2008) 55 [arXiv:0801.1590].

[2] V. Balasubramanian, A. Buchel, S.R. Green, L. Lehner and S.L. Liebling, Holographic thermalization, stability of anti-de Sitter space, and the Fermi-Pasta-Ulam paradox, Phys. Rev. Lett. 113 (2014) 071601 [arXiv: 1403.6471] [INSPIRE].

[3] J.M. Deutsch, Quantum statistical mechanics in a closed system, Phys. Rev. A 43 (1991) 2046.

[4] M. Srednicki, Chaos and quantum thermalization, Phys. Rev. E 50 (1994) 888.

[5] L.D. Landau and E.M. Lifshitz, Fluid mechanics, volume 6 of Course of theoretical physics, second edition, Butterworth-Heinemann, Oxford, U.K. (1987).

[6] J. Bhattacharya, S. Bhattacharyya, S. Minwalla and A. Yarom, A theory of first order dissipative superfluid dynamics, JHEP 05 (2014) 147 [arXiv:1105.3733] [INSPIRE].

[7] P. Kovtun, First-order relativistic hydrodynamics is stable, JHEP 10 (2019) 034 [arXiv: 1907.08191] [INSPIRE]. 
[8] J.M. Maldacena, The large $N$ limit of superconformal field theories and supergravity, Int. J. Theor. Phys. 38 (1999) 1113 [hep-th/9711200] [INSPIRE].

[9] O. Aharony, S.S. Gubser, J.M. Maldacena, H. Ooguri and Y. Oz, Large N field theories, string theory and gravity, Phys. Rept. 323 (2000) 183 [hep-th/9905111] [INSPIRE].

[10] A. Buchel, R.C. Myers and A. van Niekerk, Nonlocal probes of thermalization in holographic quenches with spectral methods, JHEP 02 (2015) 017 [Erratum ibid. 07 (2015) 137] [arXiv: 1410.6201] [INSPIRE].

[11] A. Buchel and A. Karapetyan, De Sitter vacua of strongly interacting QFT, JHEP 03 (2017) 114 [arXiv:1702.01320] [INSPIRE].

[12] A. Buchel, $\chi S B$ of cascading gauge theory in de Sitter, JHEP 05 (2020) 035 [arXiv: 1912.03566] [INSPIRE].

[13] A. Buchel, Verlinde gravity and AdS/CFT, arXiv:1702.08590 [INSPIRE].

[14] A. Buchel, Ringing in de Sitter spacetime, Nucl. Phys. B 928 (2018) 307 [arXiv: 1707.01030] [INSPIRE].

[15] A. Buchel and C. Pagnutti, Exotic hairy black holes, Nucl. Phys. B 824 (2010) 85 [arXiv: 0904.1716] [InSPIRE].

[16] A. Buchel and C. Pagnutti, Transport at criticality, Nucl. Phys. B 834 (2010) 222 [arXiv: 0912.3212] [INSPIRE].

[17] P. Bosch, A. Buchel and L. Lehner, Unstable horizons and singularity development in holography, JHEP 07 (2017) 135 [arXiv: 1704.05454] [INSPIRE].

[18] A. Buchel, Thermal order in holographic CFTs and no-hair theorem violation in black branes, Nucl. Phys. B 967 (2021) 115425 [arXiv:2005.07833].

[19] P.M. Chesler and L.G. Yaffe, Numerical solution of gravitational dynamics in asymptotically anti-de Sitter spacetimes, JHEP 07 (2014) 086 [arXiv:1309.1439] [INSPIRE].

[20] L.D. Landau and E.M. Lifshitz, Statistical physics, part 1, volume 5 of Course of theoretical physics, Butterworth-Heinemann, Oxford, U.K. (1980).

[21] A. Buchel, M.P. Heller and J. Noronha, Entropy production, hydrodynamics, and resurgence in the primordial quark-gluon plasma from holography, Phys. Rev. D 94 (2016) 106011 [arXiv: 1603.05344] [INSPIRE].

[22] A. Buchel, Entanglement entropy of $\mathcal{N}=2^{*}$ de Sitter vacuum, Nucl. Phys. B 948 (2019) 114769 [arXiv:1904.09968].

[23] A. Buchel, Singularity development and supersymmetry in holography, JHEP 08 (2017) 134 [arXiv: 1705.08560] [INSPIRE].

[24] O. Aharony, A. Buchel and P. Kerner, The black hole in the throat: thermodynamics of strongly coupled cascading gauge theories, Phys. Rev. D 76 (2007) 086005 [arXiv:0706.1768] [INSPIRE].

[25] I. Booth, Black hole boundaries, Can. J. Phys. 83 (2005) 1073 [gr-qc/0508107] [InSPIRE].

[26] P. Figueras, V.E. Hubeny, M. Rangamani and S.F. Ross, Dynamical black holes and expanding plasmas, JHEP 04 (2009) 137 [arXiv:0902.4696] [INSPIRE]. 